TRANSACTIONS OF THE

AMERICAN MATHEMATICAL SOCIETY

Volume 357, Number 8, Pages 3031-3050

S 0002-9947(04)03621-9

Article electronically published on July 16, 2004

\title{
GAUSS-MANIN CONNECTIONS FOR ARRANGEMENTS, III FORMAL CONNECTIONS
}

\author{
DANIEL C. COHEN AND PETER ORLIK
}

\begin{abstract}
We study the Gauss-Manin connection for the moduli space of an arrangement of complex hyperplanes in the cohomology of a complex rank one local system. We define formal Gauss-Manin connection matrices in the Aomoto complex and prove that, for all arrangements and all local systems, these formal connection matrices specialize to Gauss-Manin connection matrices.
\end{abstract}

\section{INTRODUCTION}

Let $\mathcal{A}=\left\{H_{1}, \ldots, H_{n}\right\}$ be an arrangement of $n$ ordered hyperplanes in $\mathbb{C}^{\ell}$, and let $\mathcal{L}$ be a local system of coefficients on $\mathrm{M}=M(\mathcal{A})=\mathbb{C}^{\ell} \backslash \bigcup_{j=1}^{n} H_{j}$, the complement of $\mathcal{A}$. The need to calculate the local system cohomology $H^{\bullet}(\mathrm{M} ; \mathcal{L})$ arises in various contexts. For instance, local systems may be used to study the Milnor fiber of the non-isolated hypersurface singularity at the origin obtained by coning the arrangement; see [8, 5]. In mathematical physics, local systems on complements of arrangements arise in the Aomoto-Gelfand theory of multivariable hypergeometric integrals [2, 12, 18] and the representation theory of Lie algebras and quantum groups. These considerations lead to solutions of the Knizhnik-Zamolodchikov differential equation from conformal field theory; see [21, 23]. Here a central problem is the determination of the Gauss-Manin connection on $H^{\bullet}(\mathrm{M} ; \mathcal{L})$ for certain discriminantal arrangements, and certain local systems.

A complex rank one local system on $\mathrm{M}$ is determined by a collection of weights $\boldsymbol{\lambda}=\left(\lambda_{1}, \ldots, \lambda_{n}\right) \in \mathbb{C}^{n}$. Associated to $\boldsymbol{\lambda}$, we have a representation $\rho: \pi_{1}(\mathrm{M}) \rightarrow$ $\mathbb{C}^{*}$, given by $\gamma_{j} \mapsto \exp \left(-2 \pi \mathrm{i} \lambda_{j}\right)$ for any meridian loop $\gamma_{j}$ about the hyperplane $H_{j}$ of $\mathcal{A}$, and an associated rank one local system $\mathcal{L}$ on $\mathrm{M}$. Parallel translation of fibers over curves in the moduli space B of all arrangements combinatorially equivalent to $\mathcal{A}$ gives rise to a Gauss-Manin connection on the vector bundle over B with fiber $H^{q}(\mathrm{M} ; \mathcal{L})$. A number of authors have considered these Gauss-Manin connections, including Aomoto [1, Schechtman and Varchenko [21, 23], Kaneko [15], and Kanarek [14.

For local systems which are nonresonant in the sense of Schechtman, Terao, and Varchenko [20, the Gauss-Manin connection matrices for general position ar-

Received by the editors July 15, 2003.

2000 Mathematics Subject Classification. Primary 32S22, 14D05, 52C35, 55N25.

Key words and phrases. Hyperplane arrangement, local system, Gauss-Manin connection.

The first author was partially supported by Louisiana Board of Regents grant LEQSF(19992002)-RD-A-01 and by National Security Agency grant MDA904-00-1-0038, and the second author was partially supported by National Security Agency grant MDA904-02-1-0019. 
rangements were found by Aomoto and Kita [2], and Terao [22] computed these connection matrices for a larger class of arrangements. For such local systems, we determined these connection matrices for all arrangements in [7]. The aim of this paper is to remove the nonresonance condition. We construct formal Gauss-Manin connection matrices in the Aomoto complex for all arrangements and prove that these formal connection matrices specialize to Gauss-Manin connection matrices.

In Section 2, we review the stratified Morse theory construction [3, 4, of a finite cochain complex $\left(K^{\bullet}(\mathcal{A}), \Delta^{\bullet}\right)$, the cohomology of which is naturally isomorphic to $H^{\bullet}(\mathrm{M} ; \mathcal{L})$. This leads to the construction of the universal complex $\left(K_{\Lambda}^{\bullet}, \Delta^{\bullet}(\mathbf{x})\right)$ for local system cohomology, where $\Lambda=\mathbb{C}\left[x_{1}^{ \pm 1}, \ldots, x_{n}^{ \pm 1}\right]$. The Orlik-Solomon algebra $A^{\bullet}$ is a finite dimensional complex vector space which models the cohomology of the complement with trivial local system, see [17, 18. The ordering of the hyperplanes of $\mathcal{A}$ provides the nbc basis for $A^{\bullet}$ over $\mathbb{C}$. Weights $\boldsymbol{\lambda}$ yield an element $a_{\boldsymbol{\lambda}}=$ $\sum \lambda_{j} a_{j}$ in $A^{1}$, and multiplication by $a_{\boldsymbol{\lambda}}$ gives $A^{\bullet}$ the structure of a cochain complex. The resulting cohomology $H^{\bullet}\left(A^{\bullet}, a_{\boldsymbol{\lambda}}\right)$ is a combinatorial analog of $H^{\bullet}(\mathrm{M} ; \mathcal{L})$. The Aomoto complex $\left(A_{R}^{\bullet}, a_{\mathbf{y}}\right)$ is the universal complex for Orlik-Solomon cohomology. Here, $A_{R}^{\bullet}$ is a free $R$-module over $A^{\bullet}$, where $R=\mathbb{C}\left[y_{1}, \ldots, y_{n}\right]$.

In Section 3, we recall the moduli space of arrangements with a fixed combinatorial type $\mathcal{T}$, following Terao 22 . It is determined by the $\operatorname{sets} \operatorname{ind}(\mathcal{T})$ and $\operatorname{dep}(\mathcal{T})$ of independent and dependent collections of $\ell+1$ element subsets of hyperplanes in $\mathcal{A}_{\infty}$, the projective closure of $\mathcal{A}$ in $\mathbb{C P}^{\ell}$. Let $\mathrm{B}(\mathcal{T})$ be a smooth, connected component of this moduli space. There is a fiber bundle $\pi: \mathrm{M}(\mathcal{T}) \rightarrow \mathrm{B}(\mathcal{T})$ whose fibers, $\pi^{-1}(\mathrm{~b})=\mathrm{M}_{\mathrm{b}}$, are complements of arrangements $\mathcal{A}_{\mathrm{b}}$ of type $\mathcal{T}$. Since $\mathrm{B}(\mathcal{T})$ is connected, $M_{b}$ is diffeomorphic to $M$.

The fiber bundle $\pi: \mathrm{M}(\mathcal{T}) \rightarrow \mathrm{B}(\mathcal{T})$ is locally trivial. Consequently, given a local system on the fiber, there is an associated flat vector bundle $\pi^{q}: \mathbf{H}^{q} \rightarrow \mathrm{B}(\mathcal{T})$, with fiber $\left(\pi^{q}\right)^{-1}(\mathrm{~b})=H^{q}\left(\mathrm{M}_{\mathrm{b}} ; \mathcal{L}_{\mathrm{b}}\right)$ at $\mathrm{b} \in \mathrm{B}(\mathcal{T})$ for each $q, 0 \leq q \leq \ell$. Fixing a basepoint $b \in \mathrm{B}(\mathcal{T})$, the operation of parallel translation of fibers over curves in $\mathrm{B}(\mathcal{T})$ in the vector bundle $\pi^{q}: \mathbf{H}^{q} \rightarrow \mathrm{B}(\mathcal{T})$ provides a complex representation $\Psi_{\mathcal{T}}^{q}: \pi_{1}(\mathrm{~B}(\mathcal{T}), \mathrm{b}) \longrightarrow \operatorname{Aut}_{\mathbb{C}}\left(H^{q}\left(\mathrm{M}_{\mathrm{b}} ; \mathcal{L}_{\mathrm{b}}\right)\right)$. The loops of primary interest are those linking moduli spaces of codimension one degenerations of $\mathcal{T}$. Such a degeneration is a type $\mathcal{T}^{\prime}$ whose moduli space $\mathrm{B}\left(\mathcal{T}^{\prime}\right)$ has codimension one in the closure of $\mathrm{B}(\mathcal{T})$. In this case, we say that $\mathcal{T}$ covers $\mathcal{T}^{\prime}$. Let $\gamma\left(\mathcal{T}^{\prime}\right) \in \pi_{1}(\mathrm{~B}(\mathcal{T})$, b) be such a loop.

The representation $\Psi_{\mathcal{T}}^{q}$ gives rise to a Gauss-Manin connection. Let $\Omega_{\mathcal{L}}^{q}\left(\mathcal{T}^{\prime}, \mathcal{T}\right)$ be a connection matrix associated to the loop $\gamma\left(\mathcal{T}^{\prime}\right)$. A key idea in this paper is to extract information about arbitrary arrangements using information about general position arrangements, of type $\mathcal{G}$, whose dependent set is empty. The moduli space $\mathrm{B}(\mathcal{G})$ of general position arrangements is the complement of a divisor $\mathrm{D}(\mathcal{G})=\bigcup_{J} \mathrm{D}_{J}$ in $\left(\mathbb{C P}^{\ell}\right)^{n}$. The components $\mathrm{D}_{J}$ of this divisor are irreducible hypersurfaces indexed by $\ell+1$ element subsets $J$ of $[n+1]$. For each such $J$, let $\mathcal{G}_{J}$ denote the combinatorial type of arrangements with $J$ as the only dependent set.

Schechtman, Terao, and Varchenko [20], refining work of Esnault, Schechtman, and Viehweg [10], found conditions on the weights which insure that the local system cohomology groups vanish except in the top dimension. These conditions depend only on the type $\mathcal{T}$, so we call weights satisfying them $\mathcal{T}$-nonresonant. Falk and Terao [1] constructed a basis for the single nonvanishing cohomology group, called the $\beta$ nbc basis. The matrices $\Omega_{\mathcal{L}}\left(\mathcal{G}_{J}, \mathcal{G}\right):=\Omega_{\mathcal{L}}^{\ell}\left(\mathcal{G}_{J}, \mathcal{G}\right)$ were computed by Aomoto and Kita [2]. 
In [7, we determined Gauss-Manin connection matrices for all pairs of arrangement types in the nonresonant case. Let $\mathcal{T}$ be a combinatorial type which covers the type $\mathcal{T}^{\prime}$. Let $\boldsymbol{\lambda}$ be a collection of $\mathcal{T}$-nonresonant weights which defines the local system $\mathcal{L}$. Weights which are $\mathcal{T}$-nonresonant are necessarily $\mathcal{G}$-nonresonant. Thus in this case, the only nonvanishing cohomology groups are in the top dimension. Since these groups depend only on the combinatorial type, we write $H^{\ell}(\mathcal{T} ; \mathcal{L})=H^{\ell}\left(\mathrm{M}_{\mathrm{b}} ; \mathcal{L}_{\mathrm{b}}\right)$ and its analog for the general position type. Define an endomorphism of $H^{\ell}(\mathcal{G} ; \mathcal{L})$ by

$$
\tilde{\Omega}_{\mathcal{L}}\left(\mathcal{T}^{\prime}, \mathcal{T}\right)=\sum_{J \in \operatorname{dep}\left(\mathcal{T}^{\prime}, \mathcal{T}\right)} m_{J}\left(\mathcal{T}^{\prime}\right) \cdot \Omega_{\mathcal{L}}\left(\mathcal{G}_{J}, \mathcal{G}\right)
$$

Here, $m_{J}\left(\mathcal{T}^{\prime}\right)$ is the order of vanishing of the restriction of a defining polynomial for $\mathrm{D}_{J}$ to $\overline{\mathrm{B}}(\mathcal{T})$ along $\mathrm{B}\left(\mathcal{T}^{\prime}\right)$, and $\operatorname{dep}\left(\mathcal{T}^{\prime}, \mathcal{T}\right)=\operatorname{dep}\left(\mathcal{T}^{\prime}\right) \backslash \operatorname{dep}(\mathcal{T})$. In 7$]$, we showed that there is a commutative diagram

$$
\begin{array}{ll}
H^{\ell}(\mathcal{G} ; \mathcal{L}) & \longrightarrow H^{\ell}(\mathcal{T} ; \mathcal{L}) \\
\quad \downarrow \tilde{\Omega}_{\mathcal{L}}\left(\mathcal{T}^{\prime}, \mathcal{T}\right) & \mid \Omega_{\mathcal{L}}\left(\mathcal{T}^{\prime}, \mathcal{T}\right) \\
H^{\ell}(\mathcal{G} ; \mathcal{L}) \longrightarrow & H^{\ell}(\mathcal{T} ; \mathcal{L})
\end{array}
$$

In particular, if $P$ is the matrix of the surjection $H^{\ell}(\mathcal{G} ; \mathcal{L}) \rightarrow H^{\ell}(\mathcal{T} ; \mathcal{L})$ in the respective $\beta$ nbc bases, then a Gauss-Manin connection matrix $\Omega_{\mathcal{L}}\left(\mathcal{T}^{\prime}, \mathcal{T}\right)$ is determined by the matrix equation

$$
P \cdot \Omega_{\mathcal{L}}\left(\mathcal{T}^{\prime}, \mathcal{T}\right)=\tilde{\Omega}_{\mathcal{L}}\left(\mathcal{T}^{\prime}, \mathcal{T}\right) \cdot P .
$$

The aim of this paper is to generalize this result to arbitrary weights. For weights $\boldsymbol{\lambda}$ which define a nontrivial local system $\mathcal{L}$, the cohomology groups $H^{q}(\mathcal{G} ; \mathcal{L})$ vanish for $q<\ell$. Thus, a statement similar to (1.1) in dimension $q<\ell$ is impossible. We solve this problem by lifting the Gauss-Manin connection to the Aomoto complex.

The Orlik-Solomon algebra and the Aomoto complex depend only on the combinatorial type, so we may label them accordingly. In Section 4 , we define for each subset $S$ of hyperplanes an endomorphism $\tilde{\omega}_{S}$ of $A_{R}^{\bullet}(\mathcal{G})$, the Aomoto complex of the general position arrangement. We show that these endomorphisms are cochain maps. In Section 5, we generalize the notion of multiplicity to all subsets of hyperplanes and the notion of dependent sets to include sets of all sizes and denote these sets $\operatorname{Dep}(\mathcal{T})$. In analogy with $\tilde{\Omega}\left(\mathcal{T}^{\prime}, \mathcal{T}\right)$, we define

$$
\tilde{\omega}\left(\mathcal{T}^{\prime}, \mathcal{T}\right)=\sum_{S \in \operatorname{Dep}\left(\mathcal{T}^{\prime}, \mathcal{T}\right)} m_{S}\left(\mathcal{T}^{\prime}\right) \cdot \tilde{\omega}_{S}
$$

In order to prove that the endomorphism $\tilde{\omega}\left(\mathcal{T}^{\prime}, \mathcal{T}\right)$ induces a cochain map $\omega\left(\mathcal{T}^{\prime}, \mathcal{T}\right)$ on $A_{R}^{\bullet}(\mathcal{T})$, the Aomoto complex of type $\mathcal{T}$, we show in Theorem 6.1 that this endomorphism preserves the subcomplex corresponding to the Orlik-Solomon ideal of this type. This provides the analog of (1.1) on the level of Aomoto complexes:

$$
\begin{aligned}
& \left(A_{R}^{\bullet}(\mathcal{G}), a_{\mathbf{y}}\right) \longrightarrow\left(A_{R}^{\bullet}(\mathcal{T}), a_{\mathbf{y}}\right) \\
& \downarrow \tilde{\omega}\left(\mathcal{T}^{\prime}, \mathcal{T}\right) \quad \downarrow \omega\left(\mathcal{T}^{\prime}, \mathcal{T}\right) \\
& \left(A_{R}^{\bullet}(\mathcal{G}), a_{\mathbf{y}}\right) \longrightarrow\left(A_{R}^{\bullet}(\mathcal{T}), a_{\mathbf{y}}\right)
\end{aligned}
$$

The horizontal maps are explicit surjections provided by the respective nbc bases. Given weights $\boldsymbol{\lambda}$, the specialization $\mathbf{y} \mapsto \boldsymbol{\lambda}$ in the chain endomorphism $\omega\left(\mathcal{T}^{\prime}, \mathcal{T}\right)$ 
defines endomorphisms $\omega_{\boldsymbol{\lambda}}^{q}\left(\mathcal{T}^{\prime}, \mathcal{T}\right): A^{q}(\mathcal{T}) \rightarrow A^{q}(\mathcal{T})$ for $0 \leq q \leq \ell$. In Section 7 we prove the main result of this paper.

Theorem. Let $\mathrm{M}$ be the complement of an arrangement of type $\mathcal{T}$ and let $\mathcal{L}$ be the local system on $\mathrm{M}$ defined by weights $\boldsymbol{\lambda}$. Suppose $\mathcal{T}$ covers $\mathcal{T}^{\prime}$. Let $\phi^{q}: K^{q} \rightarrow$ $H^{q}(\mathrm{M}, \mathcal{L})$ be the natural projection. Then there is an isomorphism $\tau^{q}: A^{q} \rightarrow$ $K^{q}$ so that a Gauss-Manin connection endomorphism $\Omega_{\mathcal{L}}^{q}\left(\mathcal{T}^{\prime}, \mathcal{T}\right)$ in local system cohomology is determined by the equation

$$
\phi^{q} \circ \tau^{q} \circ \omega_{\boldsymbol{\lambda}}^{q}\left(\mathcal{T}^{\prime}, \mathcal{T}\right)=\Omega_{\mathcal{L}}^{q}\left(\mathcal{T}^{\prime}, \mathcal{T}\right) \circ \phi^{q} \circ \tau^{q} .
$$

The groups $H^{q}(\mathrm{M} ; \mathcal{L})$ are not known in general. When these groups can be calculated explicitly, our result yields a matrix equation analogous to (1.2) which determines a Gauss-Manin connection matrix. We use the $\mathbf{n b c}$ basis for $A^{q}$ and a suitable choice of basis for $H^{q}(\mathrm{M} ; \mathcal{L})$; see Section 8 for examples.

\section{Cohomology complexes}

For an arbitrary complex local system $\mathcal{L}$ on the complement of an arrangement $\mathcal{A}$, we used stratified Morse theory in [3] to construct a complex $\left(K^{\bullet}(\mathcal{A}), \Delta^{\bullet}\right)$, the cohomology of which is naturally isomorphic to $H^{\bullet}(\mathrm{M} ; \mathcal{L})$. We recall this construction in the context of rank one local systems from [3, 4].

Let $\boldsymbol{\lambda}=\left(\lambda_{1}, \ldots, \lambda_{n}\right) \in \mathbb{C}^{n}$ be a collection of weights. Associated to $\boldsymbol{\lambda}$, we have a rank one representation $\rho: \pi_{1}(\mathrm{M}) \rightarrow \mathbb{C}^{*}$, given by $\rho\left(\gamma_{j}\right)=t_{j}$, where $\mathbf{t}=$ $\left(t_{1}, \ldots, t_{n}\right) \in\left(\mathbb{C}^{*}\right)^{n}$ is defined by $t_{j}=\exp \left(-2 \pi \mathrm{i} \lambda_{j}\right)$, and $\gamma_{j}$ is any meridian loop about the hyperplane $H_{j}$ of $\mathcal{A}$, and a corresponding rank one local system $\mathcal{L}=$ $\mathcal{L}_{\mathbf{t}}=\mathcal{L}_{\boldsymbol{\lambda}}$ on $\mathrm{M}$. Note that weights $\boldsymbol{\lambda}$ and $\boldsymbol{\lambda}^{\prime}$ yield identical representations and local systems if $\boldsymbol{\lambda}-\boldsymbol{\lambda}^{\prime} \in \mathbb{Z}^{n}$.

We assume throughout that $\mathcal{A}$ contains $\ell$ linearly independent hyperplanes. Let $\mathcal{F}$ be a complete flag (of affine subspaces) in $\mathbb{C}^{\ell}$,

$$
\mathcal{F}: \quad \emptyset=\mathcal{F}^{-1} \subset \mathcal{F}^{0} \subset \mathcal{F}^{1} \subset \mathcal{F}^{2} \subset \cdots \subset \mathcal{F}^{\ell}=\mathbb{C}^{\ell},
$$

transverse to the stratification determined by $\mathcal{A}$, so that $\operatorname{dim} \mathcal{F}^{q} \cap S_{X}=q-\operatorname{codim} S_{X}$ for each stratum, where a negative dimension indicates that $\mathcal{F}^{q} \cap S_{X}=\emptyset$. For an explicit construction of such a flag, see [3] §1]. Let $\mathrm{M}^{q}=\mathcal{F}^{q} \cap \mathrm{M}$ for each $q$. Let $K^{q}=H^{q}\left(\mathrm{M}^{q}, \mathrm{M}^{q-1} ; \mathcal{L}\right)$, and denote by $\Delta^{q}$ the boundary homomorphism $H^{q}\left(\mathrm{M}^{q}, \mathrm{M}^{q-1} ; \mathcal{L}\right) \rightarrow H^{q+1}\left(\mathrm{M}^{q+1}, \mathrm{M}^{q} ; \mathcal{L}\right)$ of the triple $\left(\mathrm{M}^{q+1}, \mathrm{M}^{q}, \mathrm{M}^{q-1}\right)$. The following compiles several results from [3].

Theorem 2.1. Let $\mathcal{L}$ be a complex rank one local system on $\mathrm{M}$.

1. For each $q, 0 \leq q \leq \ell$, we have $H^{i}\left(\mathrm{M}^{q}, \mathrm{M}^{q-1} ; \mathcal{L}\right)=0$ if $i \neq q$, and $\operatorname{dim}_{\mathbb{C}} H^{q}\left(\mathrm{M}^{q}, \mathrm{M}^{q-1} ; \mathcal{L}\right)=b_{q}(\mathcal{A})$ is equal to the $q$-th Betti number of $\mathrm{M}$ with trivial local coefficients $\mathbb{C}$.

2. The system of complex vector spaces and linear maps $\left(K^{\bullet}, \Delta^{\bullet}\right)$,

$$
K^{0} \stackrel{\Delta^{0}}{\longrightarrow} K^{1} \stackrel{\Delta^{1}}{\longrightarrow} K^{2} \longrightarrow \cdots \longrightarrow K^{\ell-1} \stackrel{\Delta^{\ell-1}}{\longrightarrow} K^{\ell},
$$

is a complex $\left(\Delta^{q+1} \circ \Delta^{q}=0\right)$. The cohomology of this complex is naturally isomorphic to $H^{\bullet}(\mathrm{M} ; \mathcal{L})$, the cohomology of $\mathrm{M}$ with coefficients in $\mathcal{L}$.

The dimensions of the terms $K^{q}$ of the complex $\left(K^{\bullet}, \Delta^{\bullet}\right)$ are independent of $\mathbf{t}$ (resp., $\boldsymbol{\lambda}, \mathcal{L}$ ). Write $\Delta^{\bullet}=\Delta^{\bullet}(\mathbf{t})$ to indicate the dependence of the complex on $\mathbf{t}$, and view these boundary maps as functions of $\mathbf{t}$. Let $\Lambda=\mathbb{C}\left[x_{1}^{ \pm 1}, \ldots, x_{n}^{ \pm 1}\right]$ be the 
ring of complex Laurent polynomials in $n$ commuting variables, and for each $q$, let $K_{\Lambda}^{q}=\Lambda \otimes_{\mathbb{C}} K^{q}$.

Theorem 2.2 ([4, Thm. 2.9]). For an arrangement $\mathcal{A}$ of $n$ hyperplanes with complement $\mathrm{M}$, there exists a universal complex $\left(K_{\Lambda}^{\bullet}, \Delta^{\bullet}(\mathbf{x})\right)$ with the following properties:

1. The terms are free $\Lambda$-modules, whose ranks are given by the Betti numbers of $\mathrm{M}, K_{\Lambda}^{q} \simeq \Lambda^{b_{q}(\mathcal{A})}$.

2. The boundary maps, $\Delta^{q}(\mathbf{x}): K_{\Lambda}^{q} \rightarrow K_{\Lambda}^{q+1}$ are $\Lambda$-linear.

3. For each $\mathbf{t} \in\left(\mathbb{C}^{*}\right)^{n}$, the specialization $\mathbf{x} \mapsto \mathbf{t}$ yields the complex $\left(K^{\bullet}, \Delta^{\bullet}(\mathbf{t})\right)$, the cohomology of which is isomorphic to $H^{\bullet}\left(\mathrm{M} ; \mathcal{L}_{\mathbf{t}}\right)$, the cohomology of $\mathrm{M}$ with coefficients in the local system associated to $\mathbf{t}$.

The entries of the boundary maps $\Delta^{q}(\mathbf{x})$ are elements of the Laurent polynomial ring $\Lambda$, the coordinate ring of the complex algebraic $n$-torus. Via the specialization $\mathbf{x} \mapsto \mathbf{t} \in\left(\mathbb{C}^{*}\right)^{n}$, we view them as holomorphic functions $\left(\mathbb{C}^{*}\right)^{n} \rightarrow \mathbb{C}$. Similarly, for each $q$, we view $\Delta^{q}(\mathbf{x})$ as a holomorphic map $\Delta^{q}:\left(\mathbb{C}^{*}\right)^{n} \rightarrow \operatorname{Mat}(\mathbb{C}), \mathbf{t} \mapsto \Delta^{q}(\mathbf{t})$.

Recall the Orlik-Solomon algebra of $\mathcal{A}, A=A(\mathcal{A})$. It is the quotient of the exterior algebra on generators $a_{j}$, for $1 \leq j \leq n$, by an ideal defined next. Given $T=\left\{j_{1}, \ldots, j_{q}\right\}$, we call $H_{j_{1}} \cap \ldots \cap H_{j_{q}}$ the intersection of $T$. We call $T$ dependent if the intersection of $T$ is not empty and $\operatorname{codim}\left(H_{j_{1}} \cap \ldots \cap H_{j_{q}}\right)<q$. Define $\partial: A(\mathcal{A}) \rightarrow A(\mathcal{A})$ by $\partial(1)=0, \partial a_{j}=1$ and for $q>1$

$$
\partial a_{T}=\sum_{k=1}^{q}(-1)^{k-1} a_{j_{1}} \ldots a_{j_{k-1}} \widehat{a_{k}} a_{j_{k+1}} \ldots a_{j_{q}} .
$$

The ideal $I(\mathcal{A})$ is generated by $\partial a_{T}$, where $T$ is dependent, and by $a_{T}$, where the intersection of $T$ is empty.

Remark 2.3. The canonical graded algebra isomorphism $H^{\bullet}(\mathrm{M}, \mathbb{C}) \simeq A^{\bullet}(\mathcal{A})$ induces an isomorphism of vector spaces $K^{q}(\mathcal{A}) \simeq A^{q}(\mathcal{A})$ for $0 \leq q \leq \ell$. This isomorphism is not canonical.

Let $a_{\boldsymbol{\lambda}}=\sum_{j=1}^{n} \lambda_{j} a_{j} \in A^{1}$. Since $a_{\boldsymbol{\lambda}} a_{\boldsymbol{\lambda}}=0,\left(A^{\bullet}, a_{\boldsymbol{\lambda}}\right)$ is a complex. There is a universal complex for the cohomology, $H^{\bullet}\left(A^{\bullet}, a_{\boldsymbol{\lambda}}\right)$. Let $R=\mathbb{C}\left[y_{1}, \ldots, y_{n}\right]$ be the polynomial ring. The Aomoto complex $\left(A_{R}^{\bullet}, a_{\mathbf{y}}\right)$ has terms $A_{R}^{q}=R \otimes_{\mathbb{C}} A^{q} \simeq R^{b_{q}(\mathcal{A})}$, and boundary maps given by $p(\mathbf{y}) \otimes \eta \mapsto \sum y_{j} p(\mathbf{y}) \otimes a_{j} \wedge \eta$. For $\boldsymbol{\lambda} \in \mathbb{C}^{n}$, the specialization $\mathbf{y} \mapsto \boldsymbol{\lambda}$ of the Aomoto complex $\left(A_{R}^{\bullet}, a_{\mathbf{y}}\right)$ yields the Orlik-Solomon complex $\left(A^{\bullet}, a_{\boldsymbol{\lambda}}\right)$. The following result was established in [4].

Theorem 2.4. For any arrangement $\mathcal{A}$, the Aomoto complex $\left(A_{R}^{\bullet}, a_{\mathbf{y}}\right)$ is chain equivalent to the linearization of the universal complex $\left(K_{\Lambda}^{\bullet}, \Delta \cdot(\mathbf{x})\right)$.

\section{Moduli SPACES}

Let $\mathcal{T}$ be the combinatorial type of the arrangement $\mathcal{A}$ of $n$ hyperplanes in $\mathbb{C}^{\ell}$ with $n \geq \ell \geq 1$. We consider the family of all arrangements of type $\mathcal{T}$. Recall that $\mathcal{A}$ is ordered by the subscripts of its hyperplanes, and we assume that $\mathcal{A}$, and hence every arrangement of type $\mathcal{T}$, contains $\ell$ linearly independent hyperplanes.

Choose coordinates $\mathbf{u}=\left(u_{1}, \ldots, u_{\ell}\right)$ on $\mathbb{C}^{\ell}$. The hyperplanes of an arrangement of type $\mathcal{T}$ are defined by linear polynomials $f_{i}=b_{i, 0}+\sum_{j=1}^{\ell} b_{i, j} u_{j}(i=1, \ldots, n)$. We embed the arrangement in projective space and add the hyperplane at infinity 
as last in the ordering, $H_{n+1}$. The moduli space of all arrangements of type $\mathcal{T}$ may be viewed as the set of matrices

$$
\mathbf{b}=\left(\begin{array}{cccc}
b_{1,0} & b_{1,1} & \cdots & b_{1, \ell} \\
b_{2,0} & b_{2,1} & \cdots & b_{2, \ell} \\
\vdots & \vdots & \ddots & \vdots \\
b_{n, 0} & b_{n, 1} & \cdots & b_{n, \ell} \\
1 & 0 & \cdots & 0
\end{array}\right),
$$

whose rows are elements of $\mathbb{C P}^{\ell}$, and whose $(\ell+1) \times(\ell+1)$ minors satisfy certain dependency conditions; see [18, Prop. 9.2.2].

Given a subset $I=\left\{i_{1}, \ldots, i_{\ell+1}\right\}$ of $[n+1]:=\{1, \ldots, n, n+1\}$, let $\Delta_{I}=\Delta_{I}(\mathrm{~b})$ denote the determinant of the submatrix of $\mathrm{b}$ with rows specified by $I$. For any combinatorial type $\mathcal{T}$, let $\operatorname{ind}(\mathcal{T})$ denote the set of all $\ell+1$ element subsets $I$ of $[n+1]$ for which $\Delta_{I} \neq 0$ in type $\mathcal{T}$. If $\mathcal{T}$ is realizable, $\operatorname{ind}(\mathcal{T})$ is the set of all subsets $I$ for which $\left\{H_{i_{1}}, \ldots, H_{i_{\ell+1}}\right\}$ is linearly independent in the projective closure of an arrangement $\mathcal{A}$ of type $\mathcal{T}$. Similarly, let $\operatorname{dep}(\mathcal{T})$ be the set of all $\ell+1$ element subsets $J$ of $[n+1]$ for which $\Delta_{J}=0$ in type $\mathcal{T}$. The moduli space of type $\mathcal{T}$ is

$$
\left\{\mathrm{b} \in\left(\mathbb{C P}^{\ell}\right)^{n} \mid \Delta_{I}(\mathrm{~b}) \neq 0 \text { for } I \in \operatorname{ind}(\mathcal{T}), \Delta_{J}(\mathrm{~b})=0 \text { for } J \in \operatorname{dep}(\mathcal{T})\right\} .
$$

Let $\mathrm{B}(\mathcal{T})$ be a smooth, connected component of this moduli space. Corresponding to each $\mathrm{b} \in \mathrm{B}(\mathcal{T})$, we have an arrangement $\mathcal{A}_{\mathrm{b}}$, combinatorially equivalent to $\mathcal{A}$, with hyperplanes defined by the first $n$ rows of the matrix equation $\mathrm{b} \cdot \tilde{\mathrm{u}}=0$, where $\tilde{\mathrm{u}}=\left(\begin{array}{llll}1 & u_{1} & \cdots & u_{\ell}\end{array}\right)^{\top}$. Let $\mathrm{M}_{\mathrm{b}}=M\left(\mathcal{A}_{\mathrm{b}}\right)$ be the complement of $\mathcal{A}_{\mathrm{b}}$. Let

$$
\mathrm{M}(\mathcal{T})=\left\{(\mathrm{b}, \mathrm{u}) \in\left(\mathbb{C P}^{\ell}\right)^{n} \times \mathbb{C}^{\ell} \mid \mathrm{b} \in \mathrm{B}(\mathcal{T}) \text { and } \mathrm{u} \in \mathrm{M}_{\mathrm{b}}\right\}
$$

and define $\pi_{\mathcal{T}}: \mathrm{M}(\mathcal{T}) \rightarrow \mathrm{B}(\mathcal{T})$ by $\pi_{\mathcal{T}}(\mathrm{b}, \mathrm{u})=\mathrm{b}$. A result of Randell [19] implies that $\pi_{\mathcal{T}}: \mathrm{M}(\mathcal{T}) \rightarrow \mathrm{B}(\mathcal{T})$ is a fiber bundle, with fiber $\pi_{\mathcal{T}}^{-1}(\mathrm{~b})=\mathrm{M}_{\mathrm{b}}$.

For each $\mathrm{b} \in \mathrm{B}(\mathcal{T})$, weights $\boldsymbol{\lambda}$ define a local system $\mathcal{L}_{\mathrm{b}}$ on $\mathrm{M}_{\mathrm{b}}$. Since $\pi_{\mathcal{T}}: \mathrm{M}(\mathcal{T}) \rightarrow$ $\mathrm{B}(\mathcal{T})$ is locally trivial, there is an associated flat vector bundle $\pi^{q}: \mathbf{H}^{q} \rightarrow \mathrm{B}(\mathcal{T})$, with fiber $\left(\pi^{q}\right)^{-1}(\mathrm{~b})=H^{q}\left(\mathrm{M}_{\mathrm{b}} ; \mathcal{L}_{\mathrm{b}}\right)$ at $\mathrm{b} \in \mathrm{B}(\mathcal{T})$ for each $q, 0 \leq q \leq \ell$. Fixing a basepoint $b \in B(\mathcal{T})$, the operation of parallel translation of fibers over curves in $\mathrm{B}(\mathcal{T})$ in the vector bundle $\pi^{q}: \mathbf{H}^{q} \rightarrow \mathrm{B}(\mathcal{T})$ provides a complex representation

$$
\Psi_{\mathcal{T}}^{q}: \pi_{1}(\mathrm{~B}(\mathcal{T}), \mathrm{b}) \longrightarrow \operatorname{Aut}_{\mathbb{C}}\left(H^{q}\left(\mathrm{M}_{\mathrm{b}} ; \mathcal{L}_{\mathrm{b}}\right)\right) .
$$

By Theorem 2.1 the local system cohomology of $M_{b}$ may be computed using the Morse-theoretic complex $K^{\bullet}\left(\mathcal{A}_{\mathrm{b}}\right)$. The fundmental group of $\mathrm{B}(\mathcal{T})$ acts by chain automorphisms on this complex, see [6, Cor. 3.2], yielding a representation

$$
\psi_{\mathcal{T}}^{\bullet}: \pi_{1}(\mathrm{~B}(\mathcal{T}), \mathrm{b}) \longrightarrow \operatorname{Aut}_{\mathbb{C}}\left(K^{\bullet}\left(\mathcal{A}_{\mathrm{b}}\right)\right) .
$$

Theorem $3.1([6])$. The representation $\Psi_{\mathcal{T}}^{q}: \pi_{1}(\mathrm{~B}(\mathcal{T}), \mathrm{b}) \rightarrow \operatorname{Aut}_{\mathbb{C}}\left(H^{q}\left(\mathrm{M}_{\mathrm{b}} ; \mathcal{L}_{\mathrm{b}}\right)\right)$ is induced by the representation $\psi_{\mathcal{T}}^{\bullet}: \pi_{1}(\mathrm{~B}(\mathcal{T}), \mathrm{b}) \rightarrow \operatorname{Aut}_{\mathbb{C}}\left(K^{\bullet}\left(\mathcal{A}_{\mathrm{b}}\right)\right)$.

The vector bundle $\pi^{q}: \mathbf{H}^{q} \rightarrow \mathrm{B}(\mathcal{T})$ supports a Gauss-Manin connection corresponding to the representation (3.2). Over a manifold $X$, there is a well known equivalence between local systems and complex vector bundles equipped with flat connections; see [9 16]. Let $\mathbf{V} \rightarrow X$ be such a bundle, with connection $\nabla$. The latter is a $\mathbb{C}$-linear map $\nabla: \mathcal{E}^{0}(\mathbf{V}) \rightarrow \mathcal{E}^{1}(\mathbf{V})$, where $\mathcal{E}^{p}(\mathbf{V})$ denotes the complex $p$-forms on $X$ with values in $\mathbf{V}$, which satisfies $\nabla(f \sigma)=\sigma d f+f \nabla(\sigma)$ for a function $f$ and $\sigma \in \mathcal{E}^{0}(\mathbf{V})$. The connection extends to a map $\nabla: \mathcal{E}^{p}(\mathbf{V}) \rightarrow \mathcal{E}^{p+1}(\mathbf{V})$ for 
$p \geq 0$, and is flat if the curvature $\nabla \circ \nabla$ vanishes. Call two connections $\nabla$ and $\nabla^{\prime}$ on $\mathbf{V}$ isomorphic if $\nabla^{\prime}$ is obtained from $\nabla$ by a gauge transformation, $\nabla^{\prime}=g \circ \nabla \circ g^{-1}$ for some $g: X \rightarrow \operatorname{Hom}(\mathbf{V}, \mathbf{V})$.

The aforementioned equivalence is given by $(\mathbf{V}, \nabla) \mapsto \mathbf{V}^{\nabla}$, where $\mathbf{V}^{\nabla}$ is the local system, or locally constant sheaf, of horizontal sections $\left\{\sigma \in \mathcal{E}^{0}(\mathbf{V}) \mid \nabla(\sigma)=0\right\}$. There is also a well known equivalence between local systems on $X$ and finite dimensional complex representations of the fundamental group of $X$. Note that isomorphic connections give rise to the same representation. Under these equivalences, the local system on $X=\mathrm{B}(\mathcal{T})$ induced by the representation $\Psi_{\mathcal{T}}^{q}$ corresponds to a flat connection on the vector bundle $\pi^{q}: \mathbf{H}^{q} \rightarrow \mathrm{B}(\mathcal{T})$, the Gauss-Manin connection.

Let $\gamma \in \pi_{1}(\mathrm{~B}(\mathcal{T}), \mathrm{b})$, and let $g: \mathbb{S}^{1} \rightarrow \mathrm{B}(\mathcal{T})$ be a representative loop. Pulling back the bundle $\pi^{q}: \mathbf{H}^{q} \rightarrow \mathrm{B}(\mathcal{T})$ and the Gauss-Manin connection $\nabla$, we obtain a flat connection $g^{*}(\nabla)$ on the vector bundle over the circle corresponding to the representation of $\pi_{1}\left(\mathbb{S}^{1}, 1\right)=\langle\zeta\rangle=\mathbb{Z}$ given by $\zeta \mapsto \Psi_{\mathcal{T}}^{q}(\gamma)$. This vector bundle is trivial since any map from the circle to the relevant classifying space is nullhomotopic. Specifying the flat connection $g^{*}(\nabla)$ amounts to choosing a logarithm of $\Psi_{\mathcal{T}}^{q}(\gamma)$. The connection $g^{*}(\nabla)$ is determined by a connection 1 -form $d z / z \otimes$ $\Omega_{\mathcal{T}}^{q}(\gamma)$, where the connection matrix $\Omega_{\mathcal{T}}^{q}(\gamma)$ corresponding to $\gamma$ satisfies $\Psi_{\mathcal{T}}^{q}(\gamma)=$ $\exp \left(-2 \pi \mathrm{i} \Omega_{\mathcal{T}}^{q}(\gamma)\right)$. If $\gamma$ and $\hat{\gamma}$ are conjugate in $\pi_{1}(\mathrm{~B}(\mathcal{T}), \mathrm{b})$, then the resulting connection matrices are conjugate, and the corresponding connections on the trivial vector bundle over the circle are isomorphic. In this sense, the connection matrix $\Omega_{\mathcal{T}}^{q}(\gamma)$ is determined by the homology class $[\gamma]$ of $\gamma$.

The loops of primary interest are those linking moduli spaces of codimension one degenerations of $\mathcal{T}$. These are types $\mathcal{T}^{\prime}$ whose moduli spaces $\mathrm{B}\left(\mathcal{T}^{\prime}\right)$ have codimension one in the closure of $\mathrm{B}(\mathcal{T})$. Define a partial order on combinatorial types as follows: $\mathcal{T} \geq \mathcal{T}^{\prime} \Longleftrightarrow \operatorname{dep}(\mathcal{T}) \subseteq \operatorname{dep}\left(\mathcal{T}^{\prime}\right)$. The combinatorial type $\mathcal{G}$ of general position arrangements is the maximal element with respect to this partial order. Write $\mathcal{T}>\mathcal{T}^{\prime}$ if $\operatorname{dep}(\mathcal{T}) \subsetneq \operatorname{dep}\left(\mathcal{T}^{\prime}\right)$. In this case we define the relative dependence set $\operatorname{dep}\left(\mathcal{T}^{\prime}, \mathcal{T}\right)=\operatorname{dep}\left(\mathcal{T}^{\prime}\right) \backslash \operatorname{dep}(\mathcal{T})$. If $\mathcal{T}>\mathcal{T}^{\prime}$, we say that $\mathcal{T}$ covers $\mathcal{T}^{\prime}$, and $\mathcal{T}^{\prime}$ is a codimension one degeneration of $\mathcal{T}$ if there is no realizable combinatorial type $\mathcal{T}^{\prime \prime}$ with $\mathcal{T}>\mathcal{T}^{\prime \prime}>\mathcal{T}^{\prime}$.

Lemma 3.2 ([7, Lemma 5.3]). The moduli space $\mathrm{B}\left(\mathcal{T}^{\prime}\right)$ has complex codimension one in the closure $\overline{\mathrm{B}}(\mathcal{T})$ of the moduli space $\mathrm{B}(\mathcal{T})$ if and only if $\mathcal{T}$ covers $\mathcal{T}^{\prime}$.

Suppose $\mathcal{T}$ covers $\mathcal{T}^{\prime}$, and let $\gamma \in \pi_{1}(\mathrm{~B}(\mathcal{T})$, b ) be a simple loop in $\mathrm{B}(\mathcal{T})$ around a generic point in $\mathrm{B}\left(\mathcal{T}^{\prime}\right)$. Denote a corresponding Gauss-Manin connection endomorphism for cohomology in the local system $\mathcal{L}$ by $\Omega_{\mathcal{L}}\left(\mathcal{T}^{\prime}, \mathcal{T}\right)$. These endomorphisms are closely related to certain combinatorial analogs defined on the Aomoto complex.

\section{Formal connections}

Let $\left.\left(A_{R}^{\bullet}(\mathcal{G}), a_{\mathbf{y}}\right)\right)$ be the Aomoto complex of a general position arrangement of $n$ hyperplanes in $\mathbb{C}^{\ell}$. Let $T=\left\{i_{1}, \ldots, i_{q}\right\}$. If order matters, then we call $T$ a $q$-tuple and write $T=\left(i_{1}, \ldots, i_{q}\right)$ and $a_{T}=a_{i_{1}} \cdots a_{i_{q}}$. Recall that $H_{n+1}$ is the hyperplane at infinity, considered the largest in the linear order. In the formulas below, a set may contain the index $n+1$ but a tuple may not. If $T=\left(i_{1}, \ldots, i_{q}\right)$ is a $q$-tuple, $1 \leq i_{k} \leq n$, then $(j, T)=\left(j, i_{1}, \ldots, i_{q}\right)$ is the $(q+1)$-tuple which adds $j$ with $1 \leq j \leq n$ to $T$ as its first entry, and $T_{k}=\left(i_{1}, \ldots, \widehat{i_{k}}, \ldots, i_{q}\right)$ is the $(q-1)$-tuple which deletes $i_{k}$ from $T$. We write $S \equiv T$ if $S$ and $T$ are equal sets. 
Definition 4.1. Let $S$ be an index set of size $q+1$. Define an $R$-linear endomorphism of the Aomoto complex, $\tilde{\omega}_{S}:\left(A_{R}^{\bullet}(\mathcal{G}), a_{\mathbf{y}}\right) \rightarrow\left(A_{R}^{\bullet}(\mathcal{G}), a_{\mathbf{y}}\right)$, as follows. In the formulas below, $T$ is a $p$-tuple, and $1 \leq j \leq n$.

If $n+1 \notin S$,

$$
\tilde{\omega}_{S}^{p}\left(a_{T}\right)= \begin{cases}y_{j} \partial a_{(j, T)} & \text { if } p=q \text { and } S \equiv(j, T), \\ a_{\mathbf{y}} \partial a_{T} & \text { if } p=q+1 \text { and } S \equiv T, \\ 0 & \text { otherwise. }\end{cases}
$$

If $n+1 \in S$,

$$
\tilde{\omega}_{S}^{p}\left(a_{T}\right)= \begin{cases}-\left(\sum_{j \in[n]-T} y_{j}\right) a_{T} & \text { if } p=q \text { and } S \equiv T \cup\{n+1\}, \\ (-1)^{k-1} y_{j} a_{\left(j, T_{k}\right)} & \text { if } p=q, S \equiv\left(j, T_{k}\right) \cup\{n+1\}, \text { and } j \notin T, \\ (-1)^{k} a_{\mathbf{y}} a_{T_{k}} & \text { if } p=q+1 \text { and } S \equiv T_{k} \cup\{n+1\}, \\ 0 & \text { otherwise. }\end{cases}
$$

Proposition 4.2. For every $S$, the map $\tilde{\omega}_{S}$ is a cochain homomorphism of the Aomoto complex $\left.\left(A_{R}^{\bullet}(\mathcal{G}), a_{\mathbf{y}}\right)\right)$.

Proof. Let $S$ be an index set of size $q+1$. Since $\tilde{\omega}_{S}^{p}=0$ for $p \neq q, q+1$, to show that $\tilde{\omega}_{S}$ is a cochain map, it suffices to check commutativity in the three squares indicated below:

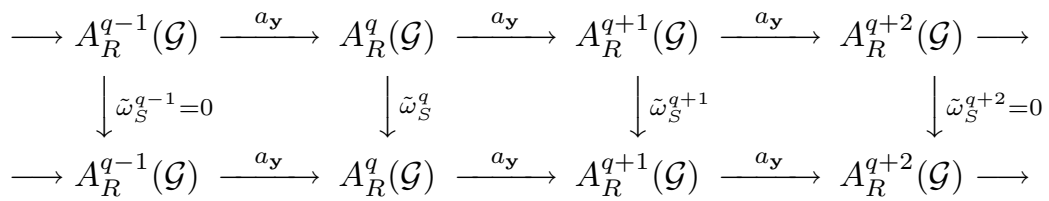

If $n+1 \notin S$, then we may assume that $S=\{1,2, \ldots, q+1\}$. Otherwise $S=$ $\{U, n+1\}$ and we may assume that $U=\{n-q+1, \ldots, n\}$. Since $A_{R}^{\bullet}(\mathcal{G})$ is free on the generators $a_{T}$, we may work with these.

In the first square above, we start with $a_{T} \in A_{R}^{q-1}$. Since $\tilde{\omega}_{S}^{q-1}=0$, we need to show that $\tilde{\omega}_{S}^{q}\left(a_{\mathbf{y}} a_{T}\right)=0$.

Suppose $n+1 \notin S$. Since $T$ must be equivalent to a subset of $S$, we may assume that $T=(3,4, \ldots, q+1)$. Then $\tilde{\omega}_{S}^{q}\left(a_{\mathbf{y}} a_{T}\right)=y_{1} y_{2}\left(\partial a_{(1,2, T)}+\partial a_{(2,1, T)}\right)=0$.

Suppose $n+1 \in S$. Since $T$ must be equivalent to a subset of $U$, we may assume $T=(n-q+2, \ldots, n)$. Then $a_{\mathbf{y}} a_{T}=\sum_{j=1}^{n-q+1} y_{j} a_{(j, T)}$. We get

$$
\begin{aligned}
\tilde{\omega}_{S}^{q}\left(a_{\mathbf{y}} a_{T}\right) & =\sum_{j=1}^{n-q} y_{j} \tilde{\omega}_{S}^{q}\left(a_{(j, T)}\right)+y_{n-q+1} \tilde{\omega}_{S}^{q}\left(a_{(n-q+1, T)}\right) \\
& =\sum_{j=1}^{n-q}(-1)^{2} y_{n-q+1} y_{j} a_{(n-q+1, j, T)_{2}}+\sum_{j=1}^{n-q}(-1) y_{j} y_{n-q+1} a_{(j, n-q+1, T)_{1}}=0 .
\end{aligned}
$$

In the second square we start with $a_{T} \in A_{R}^{q}$.

Suppose $n+1 \notin S$. Since $T$ must be equivalent to a subset of $S$, we may assume that $T=(1,2, \ldots, q)$. Then $\tilde{\omega}_{S}^{q}\left(a_{T}\right)=y_{q+1} \partial a_{(q+1, T)}$ and $a_{\mathbf{y}} \tilde{\omega}_{S}^{q}\left(a_{T}\right)=$ $y_{q+1} a_{\mathbf{y}} \partial a_{(q+1, T)}$. The only nonzero term in $\tilde{\omega}_{S}^{q+1}\left(a_{\mathbf{y}} a_{T}\right)$ is $\tilde{\omega}_{S}^{q+1}\left(y_{q+1} a_{(q+1, T)}\right)=$ $y_{q+1} a_{\mathbf{y}} \partial a_{(q+1, T)}$, so the assertion holds.

Suppose $n+1 \in S$. If $T \equiv U$, then $\tilde{\omega}_{S}^{q}\left(a_{T}\right)=(-1)^{1}\left(\sum_{j=1}^{n-q} y_{j}\right) a_{(j, T)_{1}}$ and $a_{\mathbf{y}} \tilde{\omega}_{S}^{q}\left(a_{T}\right)=-\left(\sum_{j=1}^{n-q} y_{j}\right) a_{\mathbf{y}} a_{T}$. Also, $a_{\mathbf{y}} a_{T}=\sum_{j=1}^{n-q} y_{j} a_{(j, T)}$, so $\tilde{\omega}_{S}^{q+1}\left(a_{\mathbf{y}} a_{T}\right)=$ 
$-\left(\sum_{j=1}^{n-q} y_{j}\right) a_{\mathbf{y}} a_{T}$ as required. If $T \not \equiv U$, then we may assume that $T=(n-q$, $n-q+1, \ldots, n-1)$. Then $\tilde{\omega}_{S}^{q}\left(a_{T}\right)=(-1)^{2} y_{n} a_{(n, T)_{2}}$ so $a_{\mathbf{y}} \tilde{\omega}_{S}^{q}\left(a_{T}\right)=y_{n} a_{\mathbf{y}} a_{(n, T)_{2}}$. On the other hand, there is only one term in $a_{\mathbf{y}} a_{T}$ on which $\tilde{\omega}_{S}^{q+1}$ is nonzero, namely $y_{n} a_{n} a_{T}$. Since $\tilde{\omega}_{S}^{q+1}\left(y_{n} a_{(n, T)}\right)=y_{n} a_{\mathbf{y}} a_{(n, T)_{2}}$, the assertion holds.

In the third square we start with $a_{T} \in A_{R}^{q+1}$. Since $\tilde{\omega}_{S}^{q+2}=0$, it suffices to show that $a_{\mathbf{y}} \tilde{\omega}_{S}^{q+1}\left(a_{T}\right)=0$. For any $S$, this follows from $a_{\mathbf{y}} a_{\mathbf{y}}=0$.

Remark 4.3. The map $\tilde{\omega}_{S}^{q}$ is given by geometric considerations in [2, 22, 18]. There are many possible lifts $\tilde{\omega}_{S}^{q+1}$ which make $\tilde{\omega}_{S}$ a cochain map. However, if we require that $\tilde{\omega}_{S}^{q+1}\left(a_{T}\right)=0$ unless $T$ is related to $S$ as indicated in the definition, then the lift is unique.

A collection of weights $\boldsymbol{\lambda}=\left(\lambda_{1}, \ldots, \lambda_{n}\right)$ is $\mathcal{G}$-nonresonant if $\lambda_{j} \notin \mathbb{Z}_{\geq 0}$ and $-\sum_{j=1}^{n} \lambda_{j} \notin \mathbb{Z}_{\geq 0}$. The $\beta$ nbc basis for the unique nonvanishing local system cohomology group $H^{\ell}(\mathcal{G} ; \mathcal{L})$ consists of monomials $\eta_{K}=\lambda_{k_{1}} \cdots \lambda_{k_{\ell}} a_{K}$, where $K=$ $\left(k_{1}, \ldots, k_{\ell}\right), 2 \leq k_{1}<\cdots<k_{\ell} \leq n$. In this basis, the Gauss-Manin connection is given by $\sum d \log \mathrm{D}_{J} \otimes \Omega_{\mathcal{L}}\left(\mathcal{G}_{J}, \mathcal{G}\right)$, where the sum is over all $\ell+1$ element subsets $J$ of $[n+1]$. The connection matrices $\Omega_{\mathcal{L}}\left(\mathcal{G}_{J}, \mathcal{G}\right)$ were computed in [18, recovering a result of Aomoto and Kita [2].

For $J \subset[n+1]$, let $\tilde{\omega}_{J}(\boldsymbol{\lambda})$ be the specialization $\mathbf{y} \mapsto \boldsymbol{\lambda}$ of the endomorphism $\tilde{\omega}_{J}$.

Proposition 4.4. If $\boldsymbol{\lambda}$ is a collection of $\mathcal{G}$-nonresonant weights, then for each $\ell+1$ element subset $J$ of $[n+1]$, the Gauss-Manin connection matrix $\Omega_{\mathcal{L}}\left(\mathcal{G}_{J}, \mathcal{G}\right)$ is induced by the specialization $\tilde{\omega}_{J}(\boldsymbol{\lambda})$ of the endomorphism $\tilde{\omega}_{J}$.

Proof. For $\mathcal{G}$-nonresonant weights $\boldsymbol{\lambda}$, the projection $A^{\ell}(\mathcal{G}) \rightarrow H^{\ell}(\mathcal{G} ; \mathcal{L})$ is given by

$$
a_{K} \mapsto \frac{1}{\lambda_{k_{1}} \cdots \lambda_{k_{\ell}}} \eta_{K} \text { if } 1 \notin K \text { and } a_{K} \mapsto-\frac{1}{\lambda_{k_{1}} \cdots \lambda_{k_{\ell}}} \sum_{i \notin K} \eta_{\left(i, K^{\prime}\right)} \text { if } K=\left(1, K^{\prime}\right) \text {. }
$$

For an $\ell+1$ element subset $J$ of $[n+1]$, a calculation with this projection and the endomorphism $\tilde{\omega}_{J}^{\ell}(\boldsymbol{\lambda})$ yields the result.

\section{Degenerations}

For a combinatorial type $\mathcal{T} \neq \mathcal{G}$, the Orlik-Solomon ideal $I(\mathcal{T})$ gives rise to a subcomplex $I_{R}^{\bullet}(\mathcal{T})$ of the Aomoto complex $A_{R}^{\bullet}(\mathcal{G})$, with quotient $A_{R}^{\bullet}(\mathcal{T})$. In order to prove that the endomorphisms $\tilde{\omega}\left(\mathcal{T}^{\prime}, \mathcal{T}\right)$ on $A_{R}^{\bullet}(\mathcal{G})$ induce endomorphisms $\omega\left(\mathcal{T}^{\prime}, \mathcal{T}\right)$ on $A_{R}^{\bullet}(\mathcal{T})$, we must show that they preserve this subcomplex, $\tilde{\omega}\left(\mathcal{T}^{\prime}, \mathcal{T}\right)\left(I_{R}^{\bullet}(\mathcal{T})\right) \subset$ $I_{R}^{\bullet}(\mathcal{T})$. This fact is established in the next two sections.

Definition 5.1. Given $S \subset[n+1]$, let $N_{S}(\mathcal{T})=N_{S}$ (b) denote the submatrix of (3.1) with rows specified by $S$. Let $\operatorname{rank} N_{S}(\mathcal{T})$ be the size of the largest minor with nonzero determinant. Define the multiplicity of $S$ in $\mathcal{T}$ by

$$
m_{S}(\mathcal{T})=|S|-\operatorname{rank} N_{S}(\mathcal{T}) .
$$

This definition agrees with an interpretation of multiplicity given above for $S$ with $|S|=\ell+1$. For $1<q \leq n+1$, let

$$
\operatorname{Dep}(\mathcal{T})_{q}=\left\{\left\{j_{1}, \ldots, j_{q}\right\} \mid \operatorname{codim}\left(H_{j_{1}} \cap \ldots \cap H_{j_{q}}\right)<q\right\} .
$$


The set $\operatorname{dep}(\mathcal{T})$ defined above is $\operatorname{Dep}(\mathcal{T})_{\ell+1}$. Let $\operatorname{Dep}(\mathcal{T})=\bigcup_{q} \operatorname{Dep}(\mathcal{T})_{q}$. If $\mathcal{T}>\mathcal{T}^{\prime}$, we define $\operatorname{Dep}\left(\mathcal{T}^{\prime}, \mathcal{T}\right)=\operatorname{Dep}\left(\mathcal{T}^{\prime}\right) \backslash \operatorname{Dep}(\mathcal{T})$. Let

$$
\tilde{\omega}(\mathcal{T})=\sum_{S \in \operatorname{Dep}(\mathcal{T})} m_{S}(\mathcal{T}) \cdot \tilde{\omega}_{S} \quad \text { and } \quad \tilde{\omega}\left(\mathcal{T}^{\prime}, \mathcal{T}\right)=\sum_{S \in \operatorname{Dep}\left(\mathcal{T}^{\prime}, \mathcal{T}\right)} m_{S}\left(\mathcal{T}^{\prime}\right) \cdot \tilde{\omega}_{S} .
$$

For the remainder of the paper, we fix $\mathcal{T}$ and assume that $\mathcal{T}^{\prime}$ is a codimension one degeneration of $\mathcal{T}$. Since the Aomoto complex has dimension $\ell$, only $|S| \leq \ell+1$ can contribute to the maps $\tilde{\omega}\left(\mathcal{T}^{\prime}, \mathcal{T}\right)$. A circuit is a minimally dependent set of hyperplanes. A generating set for $I(\mathcal{T})$ is obtained from the collection of circuits of $\mathcal{A}_{\infty}$.

Fix a circuit $T \in \operatorname{Dep}(\mathcal{T})$ with $|T|=q+1$. If $T=\{U, n+1\}$, then the hyperplanes of $T$ meet at infinity in $\mathcal{A}_{\infty}$, so the hyperplanes of $U$ have empty intersection in $\mathcal{A}$ and $a_{U}$ is a generator of $I(\mathcal{T})$. If $n+1 \notin T$, then $\partial a_{T}$ is a generator of $I(\mathcal{T})$. Let

$$
r_{T}= \begin{cases}a_{U} & \text { if } T=\{U, n+1\}, \\ \partial a_{T} & \text { if } n+1 \notin T .\end{cases}
$$

Note that $r_{T} \in A_{R}^{q}(\mathcal{G})$. We refer to $S \in \operatorname{Dep}\left(\mathcal{T}^{\prime}, \mathcal{T}\right)$ as $T$-relevant if $\tilde{\omega}_{S}\left(r_{T}\right) \neq 0$. For such $S$, we have $|S|=q$ or $|S|=q+1$. The next observation follows from the definition.

Lemma 5.2. Let $T$ be a q-tuple and let $S$ be any set. If $|T \cap S|<q-1$, then $\tilde{\omega}_{S}\left(a_{T}\right)=0$.

Terao 22] classified the three codimension one degeneration types in the moduli space of an arrangement whose only dependent set is the circuit $T$. In Type I, $|S \cap T| \leq q-1$ for all $S \in \operatorname{Dep}\left(\mathcal{T}^{\prime}, \mathcal{T}\right)$. By Lemma 5.2 in the case $|S \cap T|<q-1$ and a calculation in the case $|S \cap T|=q-1$, these degenerations are not $T$-relevant. In the description of the remaining two types we use the symbol $T_{p}^{m}=T_{p} \cup\{m\}$ for $1 \leq p \leq|T|$ and $m \notin T$.

II: $\left\{T_{p}^{m} \mid m \notin T\right\}$ for each fixed $p, 1 \leq p \leq|T|$,

III: $\left\{T_{p}^{m}|1 \leq p \leq| T \mid\right\}$ for each fixed $m \notin T$.

If $q=1$, then Type II does not appear.

Lemma 5.3. Let $T$ be a set of cardinality $q+1$. If $T_{i}, T_{j} \in \operatorname{Dep}(\mathcal{T})$ for $i \neq j$, then $T_{i} \in \operatorname{Dep}(\mathcal{T})$ for all $i, 1 \leq i \leq q+1$.

Proof. Without loss, assume that $T_{1}, T_{2} \in \operatorname{Dep}(\mathcal{T})$. Then there are nonzero vectors $\boldsymbol{\alpha}=\left(0, \alpha_{2}, \alpha_{3}, \ldots, \alpha_{q+1}\right)$ and $\boldsymbol{\beta}=\left(\beta_{1}, 0, \beta_{3}, \ldots, \beta_{q+1}\right)$ which are annihilated by the rows of the matrix (3.1) indexed by $T_{1}$ and $T_{2}$ respectively. If $\alpha_{i}=0$ for some $i \neq 1,2$, then $\alpha$ is annihilated by the rows corresponding to $T_{i}$; hence $T_{i} \in \operatorname{Dep}(\mathcal{T})$. If $\alpha_{i} \neq 0$, then $\alpha_{i} \boldsymbol{\beta}-\beta_{i} \boldsymbol{\alpha}$ is a nonzero vector annihilated by the rows corresponding to $T_{i}$; hence $T_{i} \in \operatorname{Dep}(\mathcal{T})$.

Proposition 5.4. Let $T \in \operatorname{Dep}(\mathcal{T})_{q+1}$ be a circuit. Then there is at most one $j \in T$ so that $T_{j} \in \operatorname{Dep}\left(\mathcal{T}^{\prime}, \mathcal{T}\right)$.

Proof. We may assume that $T=\{1, \ldots, q+1\}$. Then $T_{i}=T \backslash\{i\}$. Since $T$ is a circuit, $T_{i}$ is independent in type $\mathcal{T}$ for each $i \in T$. Since $\mathcal{T}$ is the type of an arrangement which contains $\ell$ linearly independent hyperplanes, there exists a set $J \subset[n]$ of cardinality $\ell-q$ so that $T_{1} \cup J \cup\{n+1\}$ is independent in $\mathcal{T}$. We assert that $T_{i} \cup J \cup\{n+1\}$ is independent in $\mathcal{T}$ for all $i \in T$. 
Suppose otherwise. If, for instance, $T_{q+1} \cup J \cup\{n+1\} \in \operatorname{Dep}(\mathcal{T})$, then there are constants $\alpha_{i}, \beta_{j}, \xi$, not all zero, so that

$$
\sum_{i=1}^{q} \alpha_{i} \mathrm{~b}_{i}+\sum_{j \in J} \beta_{j} \mathrm{~b}_{j}+\xi \mathrm{b}_{n+1}=0,
$$

where $\mathrm{b}_{k}$ denotes the $k$-th row of the matrix (3.1). Since $T$ is a circuit, there are constants $\zeta_{k} \neq 0$ so that $\mathrm{b}_{1}=\sum_{k=2}^{q+1} \zeta_{k} \mathrm{~b}_{k}$. Substituting this expression in (5.1) yields a dependence on the set $T_{1} \cup J \cup\{n+1\}$, which is a contradiction.

Let $S=T \cup J \cup\{n+1\}$. Then $S_{i}$ is independent in type $\mathcal{T}$ for each $i, 1 \leq i \leq q+1$. Since $\mathcal{T}^{\prime}$ is a codimension one degeneration of $\mathcal{T}$, if $T_{i} \in \operatorname{Dep}\left(\mathcal{T}^{\prime}\right)$, then $\mathrm{B}\left(\mathcal{T}^{\prime}\right)$ is locally defined by the vanishing of $\Delta_{S_{i}}$ in $\overline{\mathrm{B}}(\mathcal{T})$. If $T_{j} \in \operatorname{Dep}\left(\mathcal{T}^{\prime}\right)$ for $j \neq i$, then by Lemma $5.3 T_{k} \in \operatorname{Dep}\left(\mathcal{T}^{\prime}\right)$ for every $k$. So, as above, $\mathrm{B}\left(\mathcal{T}^{\prime}\right)$ is locally defined by the vanishing of $\Delta_{S_{k}}$ in $\overline{\mathrm{B}}(\mathcal{T})$ for every $k, 1 \leq k \leq q+1$. We will show that this is a contradiction by exhibiting a point in $\overline{\mathrm{B}}(\mathcal{T})$ for which $\Delta_{S_{1}}$ vanishes but $\Delta_{S_{k}}$ does not vanish for $k \neq 1$.

Assume that $J=\{q+2, \ldots, \ell+1\}$. Then $\mathrm{B}(\mathcal{T})$ contains points of the form

$$
\mathrm{b}(t)=\left(\begin{array}{ccc}
0 & I_{q} & 0 \\
0 & v & 0 \\
0 & 0 & I_{\ell-q} \\
F(t) & G(t) & H(t) \\
1 & 0 & 0
\end{array}\right),
$$

where $I_{k}$ is the $k \times k$ identity matrix, $v=\left(\begin{array}{llll}t & 1 & \cdots & 1\end{array}\right)$, and the submatrix $\left(\begin{array}{lll}F(t) & G(t) & H(t))\end{array}\right)$ is chosen so that $\mathrm{b}(t)$ satisfies the dependence and independence conditions of type $\mathcal{T}$ for each nonzero $t$. The point $\mathrm{b}(0)$ is in $\overline{\mathrm{B}}(\mathcal{T})$, and for $1 \leq k \leq q+1, \Delta_{S_{k}}(\mathrm{~b}(0))$ vanishes only for $k=1$. Thus, if $T_{i}, T_{j} \in \operatorname{Dep}\left(\mathcal{T}^{\prime}\right)$, there is a realizable type $\mathcal{T}^{\prime \prime}$ such that $\mathcal{T}>\mathcal{T}^{\prime \prime}>\mathcal{T}^{\prime}$, and $\mathcal{T}^{\prime}$ is a degeneration of $\mathcal{T}$ which is not of codimesion one.

This result shows that $\operatorname{Dep}\left(\mathcal{T}^{\prime}, \mathcal{T}\right)_{q}$ has no $T$-relevant element unless $T$ has a codimension one degeneration of Type II. In this case, there is a unique $p$ so that $T_{p}$ is the only $T$-relevant element in $\operatorname{Dep}\left(\mathcal{T}^{\prime}, \mathcal{T}\right)_{q}$. It remains to consider $T$-relevant $S \in \operatorname{Dep}\left(\mathcal{T}^{\prime}, \mathcal{T}\right)_{q+1}$.

Lemma 5.5. Let $T \in \operatorname{Dep}(\mathcal{T})_{q+1}$ be a circuit. If all $T$-relevant $S \in \operatorname{Dep}\left(\mathcal{T}^{\prime}, \mathcal{T}\right)_{q+1}$ belong to a family of a single type, then $m_{S}\left(\mathcal{T}^{\prime}\right)=1$ for each such $S$.

Proof. We may assume that $T=\{U, n+1\}$, where $U=\{1, \ldots, q\}$. Suppose the degeneration is of Type II, so $\{U, k\} \in \operatorname{Dep}\left(\mathcal{T}^{\prime}, \mathcal{T}\right)_{q+1}$ for some $k \in[n]-U$. We argue by contradiction. If $m_{\{U, k\}}\left(\mathcal{T}^{\prime}\right)=2$, then in type $\mathcal{T}^{\prime}$ there are two linearly independent vectors $\boldsymbol{\alpha}=\left(\alpha_{1}, \ldots, \alpha_{q}, \alpha_{k}\right)$ and $\boldsymbol{\beta}=\left(\beta_{1}, \ldots, \beta_{q}, \beta_{k}\right)$ which are annihilated by the rows of (3.1) specified by $\{U, k\}$. If $\alpha_{1}=0$, then $\left\{U_{1}, k\right\} \in$ $\operatorname{Dep}\left(\mathcal{T}^{\prime}\right)$. Since $\{U, n+1\}$ is a circuit in $\mathcal{T}$, and $\left\{U_{1}, k\right\} \notin \operatorname{Dep}(\mathcal{T})$ by Proposition 5.4, we have $\left\{U_{1}, k\right\} \in \operatorname{Dep}\left(\mathcal{T}^{\prime}, \mathcal{T}\right)$, and hence $\left\{U_{1}, k, n+1\right\} \in \operatorname{Dep}\left(\mathcal{T}^{\prime}, \mathcal{T}\right)$. This contradicts the assumption that all $T$-relevant sets $S$ belong to a Type II family. If $\alpha_{1} \neq 0$, then we use it to eliminate $\beta_{1}$ and find the same contradiction.

If the degeneration is of Type III, we may assume that $\left\{U_{1}, p, n+1\right\} \in \operatorname{Dep}\left(\mathcal{T}^{\prime}, \mathcal{T}\right)$ with $p \in[n]-U$. Assuming that $m_{\left\{U_{1}, p, n+1\right\}}\left(\mathcal{T}^{\prime}\right)=2$ leads to a similar argument. We consider the coefficient $\alpha_{n+1}$ and conclude that $\left\{U_{1}, p\right\} \in \operatorname{Dep}\left(\mathcal{T}^{\prime}, \mathcal{T}\right)$, and hence 
$\{U, p\} \in \operatorname{Dep}\left(\mathcal{T}^{\prime}, \mathcal{T}\right)$. This contradicts the assumption that all $T$-relevant sets $S$ belong to a Type III family.

Lemma 5.6. Let $T \in \operatorname{Dep}(\mathcal{T})_{q+1}$ be a circuit. Suppose $T$ gives rise to codimension one degenerations of both Type II and Type III. Then the Type II family is unique. For each Type III family there is a unique $p \in[n+1]-T$ so that $\left\{T_{i}, p\right\}$ is in both families. We call $\left\{T_{i}, p\right\}$ the intersection of these families. Moreover, $m_{\left\{T_{i}, p\right\}}\left(\mathcal{T}^{\prime}\right)=$ 2 for each intersection and $m_{S}\left(\mathcal{T}^{\prime}\right)=1$ for all other $T$-relevant $S$ in these families.

Proof. We may assume that $T=\{U, n+1\}$, where $U=\{1, \ldots, q\}$. If $T$ gives rise to a Type II family, then it is of the form $\{\{U, k\} \mid k \in[n]-U\}$. By Proposition 5.4, there is a unique $j$ for which $T_{j} \in \operatorname{Dep}\left(\mathcal{T}^{\prime}\right)_{q}$. We may assume that $j=q+1$, so that $T_{q+1}=U \in \operatorname{Dep}\left(\mathcal{T}^{\prime}\right)$. If $T$ gives rise to two different families of Type II, then also some $\left\{U_{i}, n+1\right\} \in \operatorname{Dep}\left(\mathcal{T}^{\prime}\right)$, contradicting Proposition 5.4.

Suppose there is also a Type III family involving $T$. (There may be several Type III families involving $T$, but it will be clear from the proof that we may consider one Type III family at a time.) Let $\{U, p\}$ be the intersection of the given Type II family and this Type III family. We show first that $m_{\{U, p\}}\left(\mathcal{T}^{\prime}\right)=2$. Since $U \in \operatorname{Dep}\left(\mathcal{T}^{\prime}, \mathcal{T}\right)$, it suffices to prove that row $p$ is a linear combination of the rows specified by $U$ in (3.1). Since $\{U, n+1\} \in \operatorname{Dep}(\mathcal{T})$, there is a vector $\boldsymbol{\alpha}=\left(\alpha_{1}, \ldots, \alpha_{q}, \alpha_{n+1}, 0\right)$ which is annihilated by the rows $\{U, n+1, p\}$ of (3.1). Since $\{U, n+1\}$ is a circuit, all $\alpha_{i} \neq 0$. This dependency holds also in type $\mathcal{T}^{\prime}$. Since $\left\{U_{1}, p, n+1\right\} \in \operatorname{Dep}\left(\mathcal{T}^{\prime}\right)$, we also have a vector $\boldsymbol{\beta}=\left(0, \beta_{2}, \ldots, \beta_{q}, \beta_{n+1}, \beta_{p}\right)$ annihilated by the rows $\{U, n+1, p\}$ of (3.1) in type $\mathcal{T}^{\prime}$. We claim that $\beta_{p} \neq 0$, for otherwise we would have $\left\{U_{1}, n+1\right\} \in$ $\operatorname{Dep}\left(\mathcal{T}^{\prime}, \mathcal{T}\right)$, contradicting Proposition 5.4. The vector $\beta_{n+1} \boldsymbol{\alpha}-\alpha_{n+1} \boldsymbol{\beta}$ provides the required dependence. Hence, $m_{\{U, p\}}\left(\mathcal{T}^{\prime}\right) \geq 2$. Assuming that $m_{\{U, p\}}\left(\mathcal{T}^{\prime}\right)>2$ contradicts Proposition 5.4.

The fact that the other multiplicities in these families are 1 is established as in the proof of Lemma 5.5

Theorem 5.7. The endomorphism $\tilde{\omega}\left(\mathcal{T}^{\prime}, \mathcal{T}\right)$ satisfies $\tilde{\omega}\left(\mathcal{T}^{\prime}, \mathcal{T}\right)\left(I_{R}^{\bullet}(\mathcal{T})\right) \subset I_{R}^{\bullet}(\mathcal{T})$ if and only if for each $K \subset[n]$ and each circuit $T \in \operatorname{Dep}(\mathcal{T})$, we have $\sum \tilde{\omega}_{S}\left(a_{K} r_{T}\right) \in$ $I_{R}^{\bullet}(\mathcal{T})$, where the sum is over $T$-relevant $S$ in a single type of codimension one degeneration involving $T$.

Proof. This is clear if $T$ is involved in a single type. If more types appear, then Lemma 5.6 shows that each $S$ is the intersection of at most two types. Furthermore, all such intersections have multiplicity 2 , so the corresponding $S$ may be considered individually in their respective types, each time with multiplicity 1.

\section{IDEAL INVARIANCE}

Theorem 6.1. If $\mathcal{T}$ covers $\mathcal{T}^{\prime}$, then $\tilde{\omega}\left(\mathcal{T}^{\prime}, \mathcal{T}\right)\left(I_{R}^{\bullet}(\mathcal{T})\right) \subset I_{R}^{\bullet}(\mathcal{T})$.

Proof. It follows from Theorem 5.7 that we may argue on the different types independently. It suffices to show that for every circuit $T \in \operatorname{Dep}(\mathcal{T})$, every $k$-tuple $K$, and every degeneration $\mathcal{T}^{\prime}$ of $\mathcal{T}$, we have $\tilde{\omega}\left(\mathcal{T}^{\prime}\right)\left(a_{K} r_{T}\right) \in I_{R}^{\bullet}(\mathcal{T})$. There are several cases to consider because the generators of the ideal are defined in terms of affine coordinates, while the natural action of the symmetric group on the hyperplanes is in projective space. Note that $n+1 \notin K$, and we agree to use the same symbol for the underlying set. Similarly, if $L$ is a set which does not contain $n+1$, then we write $L$ for the corresponding tuple in the natural order. 
The following identity will be useful in several places. If $J \subset[n]$, then

$$
\left(\sum_{m \in J} y_{m} a_{m}\right) \partial a_{J}=\left(\sum_{m \in J} y_{m}\right) a_{J} .
$$

Case 1. In this case, $T \in \operatorname{Dep}(\mathcal{T})$ is a circuit with $n+1 \in T$.

In this instance, $T=\{U, n+1\}$, and we may assume that $U=\{n-q+1, \ldots, n\}$. First assume $T \subset S \in \operatorname{Dep}\left(\mathcal{T}^{\prime}\right)$. Clearly, $\{K, T\} \in \operatorname{Dep}\left(\mathcal{T}^{\prime}\right)$. Let $L=[n] \backslash(K \cup U)$. We get

$$
\tilde{\omega}_{\{K, U, n+1\}}^{k+q}\left(a_{K} a_{U}\right)=-\left(\sum_{j \in L} y_{j}\right) a_{K} a_{U} .
$$

For every $j \in K,\left\{K_{j}, T\right\} \in \operatorname{Dep}\left(\mathcal{T}^{\prime}\right)$. Here

$$
\tilde{\omega}_{\left\{K_{j}, T\right\}}^{k+q}\left(a_{K} a_{U}\right)=(-1)^{j} a_{\mathbf{y}} a_{K_{j}} a_{U} .
$$

Similarly, for every $j \in K$ and every $m \in L,\left\{K_{j}^{m}, T\right\} \in \operatorname{Dep}\left(\mathcal{T}^{\prime}\right)$. Here

$$
\tilde{\omega}_{\left\{K_{j}^{m}, U, n+1\right\}}^{k+q}\left(a_{K} a_{U}\right)=a_{m} a_{K_{j}} a_{U} .
$$

In the remaining parts of this case, we may assume that $T \not \subset S$ for $S \in \operatorname{Dep}\left(\mathcal{T}^{\prime}\right)$.

Case 1.1. If $|S \cap\{K, U\}|<k+q-1$ for all other $S \in \operatorname{Dep}\left(\mathcal{T}^{\prime}\right)$, then we are done by Lemma 5.2. If there exists $S \in \operatorname{Dep}\left(\mathcal{T}^{\prime}\right)$ with $|S \cap\{K, U\}| \geq k+q-1$ and $T \not \subset S$, then $S=\left\{K, T_{p}^{m}\right\}$ with $m \in[n+1] \backslash T$. The classification implies that $T_{p}^{m}$ is in one of the remaining two types, and all the other members of that type must also be in $\operatorname{Dep}\left(\mathcal{T}^{\prime}\right)$.

Case 1.2. Suppose $T_{p}^{m}$ belongs to Type II. Then $p$ is fixed. If $p \neq q+1$, then we may assume that $p=1$. Thus $\operatorname{Dep}\left(\mathcal{T}^{\prime}\right)$ contains $S_{m}=\left\{m, K, T_{1}\right\}$ for all $m \in L$. Since every $S_{m}$ contains $F=\left\{K, T_{1}\right\}$, we conclude that $F \in \operatorname{Dep}\left(\mathcal{T}^{\prime}\right)$. Here $\tilde{\omega}_{F}^{k+q}\left(a_{K} a_{U}\right)=(-1)^{k+1} a_{\mathbf{y}} a_{K} a_{U_{1}}$ and $\tilde{\omega}_{S_{m}}^{k+q}\left(a_{K} a_{U}\right)=(-1)^{k} y_{m} a_{m} a_{K} a_{U_{1}}$. Thus

$$
\left(\tilde{\omega}_{F}^{k+q}+\sum_{m \in L} \tilde{\omega}_{S_{m}}^{k+q}\right)\left(a_{K} a_{U}\right)=-y_{n-q+1} a_{K} a_{U} .
$$

If $p=q+1$, then $\operatorname{Dep}\left(\mathcal{T}^{\prime}\right)$ contains $S_{m}=\{m, K, U\}$ for all $m \in L$. Since every $S_{m}$ contains $F=\{K, U\}$, we conclude that $F \in \operatorname{Dep}\left(\mathcal{T}^{\prime}\right)$. Here $\tilde{\omega}_{F}^{k+q}\left(a_{K} a_{U}\right)=$ $a_{\mathbf{y}} \partial\left(a_{K} a_{U}\right)$ and $\tilde{\omega}_{S_{m}}^{k+q}\left(a_{K} a_{U}\right)=y_{m} \partial a_{(m, K, U)}=y_{m} a_{K} a_{U}-y_{m} a_{m} \partial\left(a_{K} a_{U}\right)$. Thus

$$
\left(\tilde{\omega}_{F}^{k+q}+\sum_{m \in L} \tilde{\omega}_{S_{m}}^{k+q}\right)\left(a_{K} a_{U}\right)=\left(\sum_{j \in[n]} y_{j}\right) a_{K} a_{U}
$$

Case 1.3. Suppose $T_{p}^{m}$ belongs to Type III. Then $\operatorname{Dep}\left(\mathcal{T}^{\prime}\right)$ contains, for some fixed $m \in L$, the sets $S_{p}=\left\{m, K, T_{p}\right\}$ for all $p$ with $1 \leq p \leq q+1$. If $p \neq q+1$, then $\tilde{\omega}_{S_{p}}^{k+q}\left(a_{K} a_{U}\right)=(-1)^{k+p+1} y_{m} a_{(m, K, U)_{k+p+1}}=(-1)^{k+p+1} y_{m} a_{m} a_{K} a_{U_{p}}$. Thus $\sum_{p=1}^{q} \tilde{\omega}_{S_{p}}^{k+q}\left(a_{K} a_{U}\right)=(-1)^{k} y_{m} a_{m} a_{K} \partial a_{U}$. If $p=q+1$, then $\tilde{\omega}_{\{m, K, U\}}^{k+q}\left(a_{K} a_{U}\right)=$ $y_{m} \partial a_{(m, K, U)}=y_{m} a_{K} a_{U}-y_{m} a_{m}\left(\partial a_{K}\right) a_{U}+(-1)^{k+1} y_{m} a_{m} a_{K} \partial a_{U}$. Thus

$$
\sum_{p=1}^{q+1} \tilde{\omega}_{S_{p}}^{k+q}\left(a_{K} a_{U}\right)=y_{m}\left(a_{K}-a_{m} \partial a_{K}\right) a_{U} .
$$

This completes the argument in Case 1. 
Case 2. In this case, $T \in \operatorname{Dep}(\mathcal{T})$ is a circuit with $n+1 \notin T$.

In this instance, we may assume that $T=\{1, \ldots, q+1\}$. We note that $a_{T}=$ $a_{1}\left(\partial a_{T}\right) \in I(\mathcal{T})$, and hence $\partial\left(a_{K} a_{T}\right) \in I(\mathcal{T})$. First assume $T \subset S \in \operatorname{Dep}\left(\mathcal{T}^{\prime}\right)$. Clearly, $\{K, T\} \in \operatorname{Dep}\left(\mathcal{T}^{\prime}\right)$, and we have

$$
\begin{aligned}
\tilde{\omega}_{\{K, T\}}^{k+q}\left(a_{K} \partial a_{T}\right) & =\sum_{j \in T}(-1)^{j-1} \tilde{\omega}_{\{K, T\}}^{k+q}\left(a_{K} a_{T_{j}}\right)=\sum_{j \in T}(-1)^{j-1} y_{j} \partial a_{\left(j, K, T_{j}\right)} \\
& =(-1)^{k}\left(\sum_{j \in T} y_{j}\right) \partial\left(a_{K} a_{T}\right) .
\end{aligned}
$$

For $j \in K,\left\{K_{j}, T\right\} \in \operatorname{Dep}\left(\mathcal{T}^{\prime}\right)$, but $\tilde{\omega}_{\left\{K_{j}, T\right\}}^{k+q}\left(a_{K} \partial a_{T}\right)=0$. Let $L=[n] \backslash(K \cup T)$. For every $j \in K$ and every $m \in L$ we have $\left\{K_{j}^{m}, T\right\} \in \operatorname{Dep}\left(\mathcal{T}^{\prime}\right)$, but only $m=n+1$ gives a nonzero term:

$$
\tilde{\omega}_{\left\{K_{j}, T, n+1\right\}}^{k+q}\left(a_{K} \partial a_{T}\right)=(-1)^{k}\left(\sum_{s \in T} y_{s}\right) a_{K_{j}} a_{T} .
$$

In the remaining parts of this case, we may assume that $T \not \subset S$ for $S \in \operatorname{Dep}\left(\mathcal{T}^{\prime}\right)$.

Case 2.1. If $\left|S \cap\left\{K, T_{j}\right\}\right|<k+q-1$ for all other $S \in \operatorname{Dep}\left(\mathcal{T}^{\prime}\right)$ and all $j$, then we are done by Lemma 5.2 If there is a Type I degeneration $S$ with $\left|S \cap\left\{K, T_{j}\right\}\right|=k+q-1$, one can check that $n+1 \in S$, and that $\tilde{\omega}_{S}\left(a_{K} \partial a_{T}\right)=0$.

Case 2.2. Suppose $T_{p}^{m} \in \operatorname{Dep}\left(\mathcal{T}^{\prime}\right)$ belongs to Type II, where $m \in[n+1] \backslash T$. Then $p$ is fixed, and we may assume that $p=q+1$. Thus $S_{m}=\left\{m, K, T_{q+1}\right\} \in \operatorname{Dep}\left(\mathcal{T}^{\prime}\right)$ for all $m \notin T$. Since every $S_{m}$ contains $F=\left\{K, T_{q+1}\right\}$, we conclude that $F \in \operatorname{Dep}\left(\mathcal{T}^{\prime}\right)$. We have

$$
\tilde{\omega}_{F}^{k+q}\left(a_{K} \partial a_{T}\right)=(-1)^{q} a_{\mathbf{y}} \partial\left(a_{K} a_{T_{q+1}}\right) .
$$

Since $T_{q+1} \in \operatorname{Dep}\left(\mathcal{T}^{\prime}\right)$, we also have $\left\{K_{j}, T_{q+1}, n+1\right\} \in \operatorname{Dep}\left(\mathcal{T}^{\prime}\right)$ for all $j \in K$. Here $\tilde{\omega}_{\left\{K_{j}, T_{q+1}, n+1\right\}}^{k+q}\left(a_{K} \partial a_{T}\right)=(-1)^{q+j} a_{\mathbf{y}} a_{K_{j}} a_{T_{q+1}}$. Thus

$$
\sum_{j \in K} \tilde{\omega}_{\left\{K_{j}, T_{q+1}, n+1\right\}}^{k+q}\left(a_{K} \partial a_{T}\right)=(-1)^{q+1} a_{\mathbf{y}}\left(\partial a_{K}\right) a_{T_{q+1}} .
$$

If $m \neq n+1$, then $\tilde{\omega}_{\{m, F\}}^{k+q}\left(a_{K} \partial a_{T}\right)=(-1)^{q} y_{m} \partial\left(a_{m, K, T_{q+1}}\right)$. Note that $m \notin T$ by the classification, and $m \notin K$ follows from the expression. Thus $m \in L$, and we get

$$
\sum_{m \in L} \tilde{\omega}_{\{m, F\}}^{k+q}\left(a_{K} \partial a_{T}\right)=(-1)^{q} \sum_{m \in L} y_{m} \partial\left(a_{m, K, T_{q+1}}\right) .
$$

Consider $m=n+1$. We have $\tilde{\omega}_{\{F, n+1\}}^{k+q}\left(a_{K} \partial a_{T}\right)=\sum_{p=1}^{q+1}(-1)^{p-1} \tilde{\omega}_{\{F, n+1\}}^{k+q}\left(a_{K} a_{T_{p}}\right)$. For $p \neq q+1, \tilde{\omega}_{\{F, n+1\}}^{k+q}\left(a_{K} a_{T_{p}}\right)=(-1)^{p+q} y_{p} a_{K} a_{T_{q+1}}$ and $\tilde{\omega}_{\{F, n+1\}}^{k+q}\left(a_{K} a_{T_{q+1}}\right)=$ $-\left(\sum_{p \notin K \cup T_{q+1}} y_{p}\right) a_{K} a_{T_{q+1}}$. Thus

$$
\tilde{\omega}_{\{F, n+1\}}^{k+q}\left(a_{K} \partial a_{T}\right)=(-1)^{q+1}\left(\sum_{j \in[n] \backslash K} y_{j}\right) a_{K} a_{T_{q+1}} .
$$

Similarly, for every $j \in K$ and $s \in L$ we have $\left\{K_{j}^{s}, T_{q+1}, n+1\right\} \in \operatorname{Dep}\left(\mathcal{T}^{\prime}\right)$. Here $\tilde{\omega}_{\left\{K_{j}^{s}, T_{q+1}, n+1\right\}}^{k+q}\left(a_{K} a_{T_{p}}\right)=(-1)^{q+j+1} y_{s} a_{s} a_{K_{j}} a_{T_{q+1}}$. Thus

$$
\sum_{s \in L} \sum_{j \in K} \tilde{\omega}_{\left\{K_{j}^{s}, T_{q+1}, n+1\right\}}^{k+q}\left(a_{K} a_{T_{p}}\right)=(-1)^{q}\left(\sum_{s \in L} y_{s} a_{s}\right)\left(\partial a_{K}\right) a_{T_{q+1}} .
$$


Summing over all dependent sets in Type II, we must compute $\xi\left(a_{K} \partial a_{T}\right)$, where $\xi=\tilde{\omega}_{F}^{k+q}+\sum_{j \in K} \tilde{\omega}_{\left\{K_{j}, T_{q+1}, n+1\right\}}^{k+q}+\sum_{m \in L} \tilde{\omega}_{\{m, F\}}^{k+q}+\tilde{\omega}_{\{F, n+1\}}^{k+q}+\sum_{s \in L} \sum_{j \in K} \tilde{\omega}_{\left\{K_{j}^{s}, T_{q+1}, n+1\right\}}^{k+q}$.

We get

$$
\begin{aligned}
\xi\left(a_{K} \partial a_{T}\right)=( & -1)^{q} a_{\mathbf{y}} \partial\left(a_{K} a_{T_{q+1}}\right)+(-1)^{q+1} a_{\mathbf{y}}\left(\partial a_{K}\right) a_{T_{q+1}} \\
& +(-1)^{q} \sum_{m \in L} y_{m} \partial\left(a_{m, K, T_{q+1}}\right)+(-1)^{q+1}\left(\sum_{j \in[n] \backslash K} y_{j}\right) a_{K} a_{T_{q+1}} \\
& +(-1)^{q}\left(\sum_{s \in L} y_{s} a_{s}\right)\left(\partial a_{K}\right) a_{T_{q+1}} \\
= & (-1)^{q}\left(\sum_{j \in K \cup T_{q+1}} y_{j}+\sum_{m \in L} y_{m}-\sum_{j \in[n] \backslash K} y_{j}\right) a_{K} a_{T_{q+1}} \\
& +(-1)^{q}\left(y_{q+1} a_{q+1}+\sum_{m \in L} y_{m} a_{m}-\sum_{m \in L} y_{m} a_{m}\right) \partial\left(a_{K} a_{T_{q+1}}\right) \\
& \quad+(-1)^{q}\left(-\sum_{j \in[n] \backslash T_{q+1}} y_{j} a_{j}+\sum_{m \in L} y_{m} a_{m}\right)\left(\partial a_{K}\right) a_{T_{q+1}} \\
= & (-1)^{q+1} y_{q+1}\left[a_{K} a_{T_{q+1}}-a_{q+1} \partial\left(a_{K} a_{T_{q+1}}\right)+a_{q+1}\left(\partial a_{K}\right) a_{T_{q+1}}\right] \\
= & -y_{q+1} a_{K} \partial a_{T} .
\end{aligned}
$$

Case 2.3. Suppose $T_{p}^{m}$ belongs to Type III. Then $\operatorname{Dep}\left(\mathcal{T}^{\prime}\right)$ contains, for some fixed $m \notin T$, the sets $S_{p}=\left\{m, K, T_{p}\right\}$ for all $p \in T$. If $m \neq n+1$, then

$$
\sum_{p \in T} \tilde{\omega}_{S_{p}}^{k+q}\left(a_{K} \partial a_{T}\right)=\sum_{p \in T}(-1)^{p-1} y_{m} \partial\left(a_{m} a_{K} a_{T_{p}}\right)=y_{m}\left(a_{K}-a_{m} \partial a_{K}\right) \partial a_{T} .
$$

For $m=n+1$, we need the formulas

$$
\tilde{\omega}_{\left\{K, T_{p}, n+1\right\}}^{k+q}\left(a_{K} a_{T_{s}}\right)= \begin{cases}(-1)^{p+s-1} y_{s} a_{K} a_{T_{p}} & \text { if } s \neq p, \\ -\left(\sum_{j \in[n] \backslash\left\{K, T_{p}\right\}} y_{j}\right) a_{K} a_{T_{p}} & \text { if } s=p .\end{cases}
$$

We get $\tilde{\omega}_{\left\{K, T_{p}, n+1\right\}}^{k+q}\left(a_{K} \partial a_{T}\right)=(-1)^{p}\left(\sum_{j \in[n] \backslash K} y_{j}\right) a_{K} a_{T_{p}}$. Thus

$$
\sum_{p \in T} \tilde{\omega}_{\left\{K, T_{p}, n+1\right\}}^{k+q}\left(a_{K} \partial a_{T}\right)=-\left(\sum_{j \in[n] \backslash K} y_{j}\right) a_{K} \partial a_{T} .
$$

This completes the argument in Case 2, and hence the proof of Theorem 6.1.

\section{Gauss-Manin connections}

Recall the vector bundle $\pi^{q}: \mathbf{H}^{q} \rightarrow \mathrm{B}(\mathcal{T})$, with fiber $\left(\pi^{q}\right)^{-1}(\mathrm{~b})=H^{q}\left(\mathrm{M}_{\mathrm{b}} ; \mathcal{L}_{\mathrm{b}}\right)$ at $\mathrm{b} \in \mathrm{B}(\mathcal{T})$ for each $q, 0 \leq q \leq \ell$, and its Gauss-Manin connection defined in Section 3 In this section we investigate this connection and a combinatorial analog.

Let $\mathbf{A}^{q} \rightarrow \mathrm{B}(\mathcal{T})$ be the vector bundle over the moduli space whose fiber at $\mathrm{b}$ is $A^{q}\left(\mathcal{A}_{\mathrm{b}}\right)$, the $q$-th graded component of the Orlik-Solomon algebra of the arrangement $\mathcal{A}_{\mathrm{b}}$. The nbc basis provides a global trivialization of this bundle. Given weights $\boldsymbol{\lambda}$, the cohomology of the complex $\left(A^{\bullet}\left(\mathcal{A}_{\mathrm{b}}\right), a_{\boldsymbol{\lambda}}\right)$ gives rise to an additional vector bundle $\mathbf{H}^{q}(A) \rightarrow \mathrm{B}$ whose fiber at $\mathrm{b}$ is the $q$-th cohomology group of the Orlik-Solomon algebra, $H^{q}\left(A^{\bullet}\left(\mathcal{A}_{\mathrm{b}}\right), a_{\boldsymbol{\lambda}}\right)$. Like their topological counterparts, these combinatorial vector bundles admit flat connections, which we call combinatorial Gauss-Manin connections; see [6, §5]. 
Theorem 6.1 implies that we have a commutative diagram of cochain complexes

$$
\begin{aligned}
& \left(I_{R}^{\bullet}(\mathcal{T}), a_{\mathbf{y}}\right) \stackrel{\iota}{\longrightarrow}\left(A_{R}^{\bullet}(\mathcal{G}), a_{\mathbf{y}}\right) \stackrel{p}{\longrightarrow}\left(A_{R}^{\bullet}(\mathcal{T}), a_{\mathbf{y}}\right) \\
& \left.\downarrow \tilde{\omega}\left(\mathcal{T}^{\prime}, \mathcal{T}\right)\right|_{I_{R}^{\bullet}(\mathcal{T})} \quad \downarrow \tilde{\omega}\left(\mathcal{T}^{\prime}, \mathcal{T}\right) \quad \downarrow \omega\left(\mathcal{T}^{\prime}, \mathcal{T}\right) \\
& \left(I_{R}^{\bullet}(\mathcal{T}), a_{\mathbf{y}}\right) \stackrel{\iota}{\longrightarrow}\left(A_{R}^{\bullet}(\mathcal{G}), a_{\mathbf{y}}\right) \stackrel{p}{\longrightarrow}\left(A_{R}^{\bullet}(\mathcal{T}), a_{\mathbf{y}}\right)
\end{aligned}
$$

where $\iota: I_{R}^{\bullet}(\mathcal{T}) \rightarrow A_{R}^{\bullet}(\mathcal{G})$ is the inclusion, $p: A_{R}^{\bullet}(\mathcal{G}) \rightarrow A_{R}^{\bullet}(\mathcal{T})$ is the projection provided by the respective nbc bases, and $\omega\left(\mathcal{T}^{\prime}, \mathcal{T}\right)$ is the induced map. Given weights $\boldsymbol{\lambda}$, the specialization $\mathbf{y} \mapsto \boldsymbol{\lambda}$ in the chain endomorphism $\omega\left(\mathcal{T}^{\prime}, \mathcal{T}\right)$ defines endomorphisms $\omega_{\lambda}^{q}\left(\mathcal{T}^{\prime}, \mathcal{T}\right): A^{q}(\mathcal{T}) \rightarrow A^{q}(\mathcal{T})$ for $0 \leq q \leq \ell$.

Theorem 7.1. Let $\mathrm{M}$ be the complement of an arrangement of type $\mathcal{T}$ and let $\mathcal{L}$ be the local system on $\mathrm{M}$ defined by weights $\boldsymbol{\lambda}$. Suppose $\mathcal{T}$ covers $\mathcal{T}^{\prime}$.

1. Let $\rho^{q}: A^{q} \rightarrow H^{q}\left(A^{\bullet}(\mathcal{T}), a_{\boldsymbol{\lambda}}\right)$ be the natural projection. Then a combinatorial Gauss-Manin connection endomorphism $\Omega_{\mathcal{C}}^{q}\left(\mathcal{T}^{\prime}, \mathcal{T}\right)$ in Orlik-Solomon algebra cohomology is determined by the equation

$$
\rho^{q} \circ \omega_{\boldsymbol{\lambda}}^{q}\left(\mathcal{T}^{\prime}, \mathcal{T}\right)=\Omega_{\mathcal{C}}^{q}\left(\mathcal{T}^{\prime}, \mathcal{T}\right) \circ \rho^{q} .
$$

2. Let $\phi^{q}: K^{q} \rightarrow H^{q}(\mathrm{M}, \mathcal{L})$ be the natural projection. Then there is an isomorphism $\tau^{q}: A^{q} \rightarrow K^{q}$ so that a Gauss-Manin connection endomorphism $\Omega_{\mathcal{L}}^{q}\left(\mathcal{T}^{\prime}, \mathcal{T}\right)$ in local system cohomology is determined by the equation

$$
\phi^{q} \circ \tau^{q} \circ \omega_{\boldsymbol{\lambda}}^{q}\left(\mathcal{T}^{\prime}, \mathcal{T}\right)=\Omega_{\mathcal{L}}^{q}\left(\mathcal{T}^{\prime}, \mathcal{T}\right) \circ \phi^{q} \circ \tau^{q} .
$$

Proof. For $\mathcal{T}$-nonresonant weights, it is known that $H^{q}\left(\mathrm{M}_{\mathrm{b}} ; \mathcal{L}_{\mathrm{b}}\right) \simeq H^{q}\left(A^{\bullet}\left(\mathcal{A}_{\mathrm{b}}\right), a_{\boldsymbol{\lambda}}\right)$ for all $q\left[20\right.$. Moreover, a Gauss-Manin connection matrix $\Omega_{\mathcal{L}}^{\ell}\left(\mathcal{T}^{\prime}, \mathcal{T}\right)=\Omega_{\mathcal{C}}^{\ell}\left(\mathcal{T}^{\prime}, \mathcal{T}\right)$ in the $\beta \mathbf{n b c}$ basis for the unique nonvanishing local system cohomology group is induced by the endomorphism $\tilde{\Omega}_{\mathcal{L}}\left(\mathcal{T}^{\prime}, \mathcal{T}\right)$ of the top cohomology of the complement of a general position arrangement by [7, Thm. 7.3], see also (1.2). In turn, $\tilde{\Omega}_{\mathcal{L}}\left(\mathcal{T}^{\prime}, \mathcal{T}\right)$ is induced by the specialization $\tilde{\omega}_{\boldsymbol{\lambda}}\left(\mathcal{T}^{\prime}, \mathcal{T}\right)$ of the endomorphism $\tilde{\omega}\left(\mathcal{T}^{\prime}, \mathcal{T}\right)$ of the Aomoto complex of a general position arrangement, see Proposition 4.4 Hence, it follows from Theorem6.1 that $\Omega_{\mathcal{L}}^{\ell}\left(\mathcal{T}^{\prime}, \mathcal{T}\right)$ is induced by the specialization $\omega_{\boldsymbol{\lambda}}\left(\mathcal{T}^{\prime}, \mathcal{T}\right)$ of the endomorphism $\omega\left(\mathcal{T}^{\prime}, \mathcal{T}\right)$ of the Aomoto complex of type $\mathcal{T}$.

Thus for $\mathcal{T}$-nonresonant weights, the endomorphism $\omega\left(\mathcal{T}^{\prime}, \mathcal{T}\right)$ induces both the Gauss-Manin connection matrix and its combinatorial analog in the $\beta \mathbf{n b c}$ basis. Since $\omega\left(\mathcal{T}^{\prime}, \mathcal{T}\right)$ is a holomorphic map in the variables $\mathbf{y}$ and the set of $\mathcal{T}$-nonresonant weights is open and dense, $\omega\left(\mathcal{T}^{\prime}, \mathcal{T}\right)$ induces a Gauss-Manin connection endomorphism for all weights in either cohomology theory.

\section{An EXAmple}

Let $\mathcal{T}$ be the combinatorial type of the arrangement $\mathcal{A}$ of 4 lines in $\mathbb{C}^{2}$ depicted in Figure 1 Here $\mathrm{B}(\mathcal{T})$ is codimension one in $\left(\mathbb{C P}^{2}\right)^{4}=\overline{\mathrm{B}}(\mathcal{G})$.
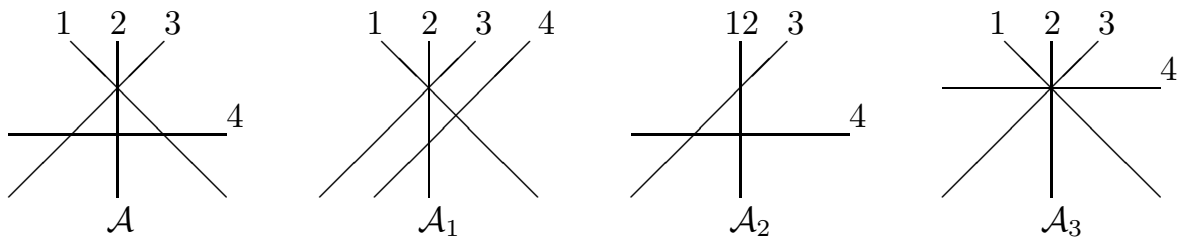

Figure 1. A codimension one arrangement and three degenerations 
For $\mathcal{T}$-nonresonant weights $\boldsymbol{\lambda}$, Gauss-Manin connection matrices $\Omega_{\mathcal{L}}^{2}\left(\mathcal{T}^{\prime}, \mathcal{T}\right)$ were determined by Terao [22, 18]. These calculations were recovered in [7], and may also be obtained using the methods of this paper.

For a nontrivial resonant local system $\mathcal{L}$ on the complement $\mathrm{M}$ of $\mathcal{A}$, we used ad hoc methods in [6, §6] to determine a Gauss-Manin connection matrix $\Omega_{\mathcal{L}}^{2}\left(\mathcal{T}_{2}, \mathcal{T}\right)$, where $\mathcal{T}_{2}$ is the combinatorial type of the codimension one degeneration $\mathcal{A}_{2}$ of $\mathcal{A}$ shown in Figure 1. The results of the previous sections may be used to obtain these connection matrices for all codimension one degenerations. We illustrate this by means of representative examples.

The universal complex $\left(K_{\Lambda}^{\bullet}, \Delta^{\bullet}(\mathbf{x})\right)$ and Aomoto complex $\left(A_{R}^{\bullet}, a_{\mathbf{y}}\right)$ of $\mathcal{A}$ are recorded in [6, $\S 6.1]$. In particular, the coboundary maps $\Delta^{1}(\mathbf{x}): K_{\Lambda}^{1} \rightarrow K_{\Lambda}^{2}$ and $a_{\mathbf{y}}: A_{R}^{1} \rightarrow A_{R}^{2}$ have matrices

$$
\Delta^{1}=\Delta^{1}(\mathbf{x})=\left[\begin{array}{ccccc}
x_{3}-x_{2} x_{3} & 1-x_{3} & 1-x_{4} & 0 & 0 \\
x_{1} x_{3}-1 & x_{1}-x_{1} x_{3} & 0 & 1-x_{4} & 0 \\
1-x_{2} & x_{1} x_{2}-1 & 0 & 0 & 1-x_{4} \\
0 & 0 & x_{1}-1 & x_{2}-1 & x_{3}-1
\end{array}\right]
$$

and

$$
\mu^{1}=\mu^{1}(\mathbf{y})=\left[\begin{array}{ccccc}
-y_{2} & -y_{3} & -y_{4} & 0 & 0 \\
y_{1}+y_{3} & -y_{3} & 0 & -y_{4} & 0 \\
-y_{2} & y_{1}+y_{2} & 0 & 0 & -y_{4} \\
0 & 0 & y_{1} & y_{2} & y_{3}
\end{array}\right]
$$

The combinatorial types $\mathcal{T}_{i}$ of the (multi)-arrangements $\mathcal{A}_{i}$ shown in Figure 1 are codimension one degenerations of $\mathcal{T}$, corresponding to irreducible components of the divisor $\mathrm{D}(\mathcal{T})=\overline{\mathrm{B}}(\mathcal{T}) \backslash \mathrm{B}(\mathcal{T})$. For these degenerations, we have

$\operatorname{Dep}\left(\mathcal{T}_{1}, \mathcal{T}\right)=\{345\}, \operatorname{Dep}\left(\mathcal{T}_{2}, \mathcal{T}\right)=\{12,124,125\}, \operatorname{Dep}\left(\mathcal{T}_{3}, \mathcal{T}\right)=\{124,134,234\}$

The corresponding endomorphisms $\tilde{\omega}\left(\mathcal{T}_{i}, \mathcal{T}\right)$ of the Aomoto complex $A_{R}(\mathcal{G})$ of a general position arrangement of four lines are given by

$\tilde{\omega}\left(\mathcal{T}_{1}, \mathcal{T}\right)=\tilde{\omega}_{345}, \quad \tilde{\omega}\left(\mathcal{T}_{2}, \mathcal{T}\right)=\tilde{\omega}_{12}+\tilde{\omega}_{124}+\tilde{\omega}_{125}, \quad \tilde{\omega}\left(\mathcal{T}_{3}, \mathcal{T}\right)=\tilde{\omega}_{124}+\tilde{\omega}_{134}+\tilde{\omega}_{234}$.

Let $p: A^{\bullet}(\mathcal{G}) \rightarrow A^{\bullet}(\mathcal{T})$ be the natural projection, given in the nbc bases by

$$
p\left(a_{J}\right)= \begin{cases}a_{1,3}-a_{1,2} & \text { if } J=\{2,3\} \\ a_{J} & \text { otherwise. }\end{cases}
$$

By Theorem 6.1 the chain endomorphisms $\tilde{\omega}\left(\mathcal{T}_{j}, \mathcal{T}\right)$ of $A^{\bullet}(\mathcal{G})$ induce chain endomorphisms $\omega\left(\mathcal{T}_{j}, \mathcal{T}\right)$ of $A^{\bullet}(\mathcal{T})$. A calculation with the projection $p$ reveals that these are given by $\omega^{1}\left(\mathcal{T}_{j}, \mathcal{T}\right)=\tilde{\omega}^{1}\left(\mathcal{T}_{j}, \mathcal{T}\right)$ for each $j$, and

$$
\begin{aligned}
\omega^{2}\left(\mathcal{T}_{1}, \mathcal{T}\right)= & {\left[\begin{array}{llllc}
0 & 0 & 0 & 0 & 0 \\
0 & 0 & 0 & 0 & -y_{4} \\
0 & 0 & 0 & 0 & y_{3} \\
0 & 0 & 0 & 0 & y_{3} \\
0 & 0 & 0 & 0 & -y_{1}-y_{2}
\end{array}\right], \quad \omega^{2}\left(\mathcal{T}_{2}, \mathcal{T}\right)=\left[\begin{array}{cccccc}
y_{1}+y_{2} & 0 & 0 & 0 & 0 \\
y_{2} & 0 & 0 & 0 & 0 \\
0 & 0 & y_{2} & -y_{2} & 0 \\
0 & 0 & -y_{1} & y_{1} & 0 \\
0 & 0 & 0 & 0 & 0
\end{array}\right] } \\
\omega^{2}\left(\mathcal{T}_{3}, \mathcal{T}\right) & =\left[\begin{array}{ccccc}
y_{4} & 0 & -y_{4} & y_{4} & 0 \\
0 & y_{4} & -y_{4} & 0 & y_{4} \\
-y_{2} & -y_{3} & y_{2}+y_{3} & -y_{2} & -y_{3} \\
y_{1}+y_{3} & -y_{3} & -y_{1} & y_{1}+y_{3} & -y_{3} \\
-y_{2} & y_{1}+y_{2} & -y_{1} & -y_{2} & y_{1}+y_{2}
\end{array}\right]
\end{aligned}
$$


8.1. Nonresonant weights. For $\mathcal{T}$-nonresonant weights, local system cohomology is isomorphic to the cohomology of the Orlik-Solomon complex. So it suffices to find combinatorial Gauss-Manin connection matrices in this instance.

Weights $\boldsymbol{\lambda}$ are $\mathcal{T}$-nonresonant if $\lambda_{1}, \lambda_{2}, \lambda_{3}, \lambda_{4}, \lambda_{\{1,2,3\}},-\lambda_{[4]} \notin \mathbb{Z}_{\geq 0}$, where $\lambda_{J}=$ $\sum_{j \in J} \lambda_{j}$ for a set $J$. The $\beta$ nbc basis for $H^{2}\left(A^{\bullet}(\mathcal{T}), a_{\boldsymbol{\lambda}}\right)$ is $\left\{\eta_{2,4}, \eta_{3,4}\right\}$, where $\eta_{i, j}=\lambda_{i} \lambda_{j} a_{i, j}$, and the projection $\rho^{2}: A^{2}(\mathcal{T}) \rightarrow H^{2}\left(A^{\bullet}(\mathcal{T}), a_{\boldsymbol{\lambda}}\right)$ is given by

$$
\rho^{2}\left(a_{i, j}\right)= \begin{cases}\left(\lambda_{\{1,2\}} \eta_{2,4}+\lambda_{2} \eta_{3,4}\right) /\left(\lambda_{1} \lambda_{2} \lambda_{\{1,2,3\}}\right) & \text { if }\{i, j\}=\{1,2\}, \\ \left(\lambda_{3} \eta_{2,4}+\lambda_{\{1,3\}} \eta_{3,4}\right) /\left(\lambda_{1} \lambda_{3} \lambda_{\{1,2,3\}}\right) & \text { if }\{i, j\}=\{1,3\}, \\ -\left(\eta_{2,4}+\eta_{3,4}\right) /\left(\lambda_{1} \lambda_{4}\right) & \text { if }\{i, j\}=\{1,4\}, \\ \eta_{i, j} /\left(\lambda_{i} \lambda_{j}\right) & \text { if }\{i, j\}=\{2,4\} \text { or }\{3,4\} .\end{cases}
$$

By Theorem [7.1, connection matrices corresponding to the codimension one degenerations $\mathcal{T}_{j}$ of $\mathcal{T}$ satisfy $\rho^{2} \circ \omega_{\boldsymbol{\lambda}}^{2}\left(\mathcal{T}_{j}, \mathcal{T}\right)=\Omega_{\mathcal{C}}^{2}\left(\mathcal{T}_{j}, \mathcal{T}\right) \circ \rho^{2}$. Calculations with the endomorphisms $\omega_{\boldsymbol{\lambda}}^{2}\left(\mathcal{T}_{j}, \mathcal{T}\right)=\left.\omega^{2}\left(\mathcal{T}_{j}, \mathcal{T}\right)\right|_{\mathbf{y} \mapsto \boldsymbol{\lambda}}$ and the projection $\rho^{2}$ yield

$$
\Omega_{\mathcal{C}}^{2}\left(\mathcal{T}_{1}, \mathcal{T}\right)=\left[\begin{array}{cc}
0 & \lambda_{2} \\
0 & -\lambda_{\{1,2\}}
\end{array}\right], \Omega_{\mathcal{C}}^{2}\left(\mathcal{T}_{2}, \mathcal{T}\right)=\left[\begin{array}{cc}
\lambda_{\{1,2\}} & \lambda_{2} \\
0 & 0
\end{array}\right], \Omega_{\mathcal{C}}^{2}\left(\mathcal{T}_{3}, \mathcal{T}\right)=\left[\begin{array}{cc}
\lambda_{[4]} & 0 \\
0 & \lambda_{[4]}
\end{array}\right],
$$

compare [18 Ex. 10.4.2] and [7, Ex. 8.2]. Similar calculations recover the remaining connection matrices recorded in [18, Ex. 10.4.2].

8.2. Resonant weights. A nontrivial resonant local system $\mathcal{L}$ on $\mathrm{M}$ corresponds to a point $\mathbf{1} \neq \mathbf{t} \in\left(\mathbb{C}^{*}\right)^{4}$ satisfying $t_{1} t_{2} t_{3}=1$ and $t_{4}=1$. For each such $\mathbf{t}$, we have $H^{2}(\mathrm{M} ; \mathcal{L}) \simeq \mathbb{C}^{3}$. Define $\Xi: \Lambda^{5} \rightarrow \Lambda^{3}$ and $\Upsilon: R^{5} \rightarrow R^{3}$ by

$$
\Xi=\left[\begin{array}{ccc}
x_{1} x_{2}-1 & 0 & x_{1} x_{2}+x_{4}-2 \\
x_{2}-1 & 0 & x_{2}-1 \\
0 & x_{2}-1 & 0 \\
0 & 1-x_{1} & x_{3}-1 \\
0 & 0 & 1-x_{2}
\end{array}\right]
$$

and

$$
\Upsilon=\left[\begin{array}{ccc}
y_{1}+y_{2} & 0 & y_{1}+y_{2}+y_{4} \\
y_{2} & 0 & y_{2} \\
0 & y_{2} & 0 \\
0 & -y_{1} & y_{3} \\
0 & 0 & -y_{2}
\end{array}\right]
$$

Note that $\Upsilon$ is the linearization of $\Xi$. Since $\mathcal{L}$ is nontrivial, $t_{i} \neq 1$ for some $i$. Assume, without loss, that $t_{2} \neq 1$. For each $\mathbf{t}$ satisfying $t_{1} t_{2} t_{3}=1, t_{4}=1$, and this condition, check that $\operatorname{rank} \Xi(\mathbf{t})=3$ and $\Xi(\mathbf{t}) \circ \Delta^{1}(\mathbf{t})=0$. So the projection $\mathbb{C}^{5} \simeq K^{2} \rightarrow H^{2}(\mathrm{M} ; \mathcal{L}) \simeq \mathbb{C}^{3}$ may be realized as the evaluation $\phi^{2}=\Xi(\mathbf{t})$.

Via the map $\Upsilon$, the endomorphisms $\omega\left(\mathcal{T}_{j}, \mathcal{T}\right)$ induce maps $\Omega_{j}: R^{3} \rightarrow R^{3}$, which satisfy $\Upsilon \circ \omega^{2}\left(\mathcal{T}_{j}, \mathcal{T}\right)=\Omega_{j} \circ \Upsilon$. Writing $y_{J}=\sum_{j \in J} y_{j}$, these maps have matrices

$$
\Omega_{1}=\left[\begin{array}{ccc}
0 & 0 & y_{\{1,2,4\}} \\
0 & 0 & -y_{3} \\
0 & 0 & -y_{\{1,2\}}
\end{array}\right], \Omega_{2}=\left[\begin{array}{ccc}
y_{\{1,2\}} & 0 & y_{\{1,2,4\}} \\
0 & y_{\{1,2\}} & -y_{3} \\
0 & 0 & 0
\end{array}\right], \Omega_{3}=\left[\begin{array}{ccc}
y_{4} & -y_{4} & -y_{[4]} \\
-y_{[3]} & y_{[3]} & -y_{[4]} \\
0 & 0 & y_{[4]}
\end{array}\right] .
$$

Let $\boldsymbol{\lambda}$ be a collection of weights corresponding to $\mathbf{t}$. Note that $\lambda_{2} \notin \mathbb{Z}$ since $t_{2} \neq 1$. Hence, $\Upsilon(\boldsymbol{\lambda}): \mathbb{C}^{5} \rightarrow \mathbb{C}^{3}$ is surjective for all such $\boldsymbol{\lambda}$. Identify $H^{2}(\mathrm{M} ; \mathcal{L})=\mathbb{C}^{3}$ and let $\tau^{2}: A^{2} \rightarrow K^{2}$ be an isomorphism for which $\phi^{2} \circ \tau^{2}=\Upsilon(\boldsymbol{\lambda})$. Theorem 7.1 implies that a Gauss-Manin connection matrix $\Omega_{\mathcal{L}}^{2}\left(\mathcal{T}_{j}, \mathcal{T}\right)$ in $H^{2}(\mathrm{M} ; \mathcal{L})$ corresponding to the degeneration $\mathcal{T}_{j}$ of $\mathcal{T}$ satisfies $\Upsilon(\boldsymbol{\lambda}) \cdot \omega_{\boldsymbol{\lambda}}^{q}\left(\mathcal{T}^{\prime}, \mathcal{T}\right)=\Omega_{\mathcal{L}}^{q}\left(\mathcal{T}_{j}, \mathcal{T}\right) \cdot \Upsilon(\boldsymbol{\lambda})$. Since the 
equality $\Upsilon \circ \omega^{2}\left(\mathcal{T}_{j}, \mathcal{T}\right)=\Omega_{j} \circ \Upsilon$ holds in the Aomoto complex, the specialization $\mathbf{y} \mapsto \boldsymbol{\lambda}$ yields connection matrices $\Omega_{\mathcal{L}}^{2}\left(\mathcal{T}_{j}, \mathcal{T}\right)=\Omega_{j}(\boldsymbol{\lambda})$.

The endomorphisms $\Omega_{\mathcal{L}}^{1}\left(\mathcal{T}_{j}, \mathcal{T}\right)$ may be determined by similar methods. As noted in [6], the endomorphism $\Omega_{\mathcal{L}}^{1}\left(\mathcal{T}_{2}, \mathcal{T}\right)$ corresponds to the automorphism of $H^{1}(\mathrm{M} ; \mathcal{L}) \simeq \mathbb{C}$ given by multiplication by $t_{1} t_{2}$, so has matrix $\left[\lambda_{\{1,2\}}\right]$. (Note that $\lambda_{\{1,2\}} \in \mathbb{Z}$ if $t_{1} t_{2}=1$.) The endomorphisms $\Omega_{\mathcal{L}}^{1}\left(\mathcal{T}_{1}, \mathcal{T}\right)$ and $\Omega_{\mathcal{L}}^{1}\left(\mathcal{T}_{3}, \mathcal{T}\right)$ are trivial.

Connection matrices $\Omega_{\mathcal{L}}^{q}\left(\mathcal{T}^{\prime}, \mathcal{T}\right)$ corresponding to other codimension one degenerations of $\mathcal{T}$ may be obtained by analogous calculations. Note however that the projection $\Upsilon(\boldsymbol{\lambda}): A^{2} \rightarrow H^{2}(\mathrm{M} ; \mathcal{L})$ need not be relevant for all degenerations.

\section{REFERENCES}

[1] K. Aomoto, Gauss-Manin connection of integral of difference products, J. Math. Soc. Japan 39 (1987), 191-208. MR 88f:32031

[2] K. Aomoto, M. Kita, Hypergeometric Functions (in Japanese), Springer-Verlag, Tokyo, 1994.

[3] D. Cohen, Cohomology and intersection cohomology of complex hyperplane arrangements, Adv. Math. 97 (1993), 231-266. MR 94a:32055

[4] D. Cohen, P. Orlik, Arrangements and local systems, Math. Res. Lett. 7 (2000), 299-316. MR 2001i:57040

[5] - Some cyclic covers of complements of arrangements, Topology Appl. 118 (2002), 3-15. MR 2003h:32039

[6] _ Gauss-Manin connections for arrangements, I. Eigenvalues, Compositio Math. 136 (2003), 299-316. MR 2004a:32042

[7] —, Gauss-Manin connections for arrangements, II. Nonresonant weights, Amer. J. Math., to appear; math.AG/0207114.

[8] D. Cohen, A. Suciu, On Milnor fibrations of arrangements, J. London Math. Soc. 51 (1995), 105-119. MR 96e:32034

[9] P. Deligne, Equations Différentielles à Points Singuliers Réguliers, Lect. Notes in Math., vol. 163, Springer-Verlag, Berlin-New York, 1970. MR 54:5232

[10] H. Esnault, V. Schechtman, V. Viehweg, Cohomology of local systems on the complement of hyperplanes, Invent. Math. 109 (1992), 557-561; Erratum, ibid. 112 (1993), 447. MR 93g:32051; MR 94b:32061

[11] M. Falk, H. Terao, $\beta$ nbc-bases for cohomology of local systems on hyperplane complements, Trans. Amer. Math. Soc. 349 (1997), 189-202. MR 97g:52029

[12] I. Gelfand, General theory of hypergeometric functions, Soviet Math. Dokl. 33 (1986), 573577. MR 87h:22012

[13] M. Goresky, R. MacPherson, Stratified Morse Theory, Ergeb. Math. Grenzgeb., vol. 14, Springer-Verlag, Berlin-New York, 1988. MR 90d:57039

[14] H. Kanarek, Gauss-Manin connection arising from arrangements of hyperplanes, Illinois J. Math. 44 (2000), 741-766. MR 2002m:14006

[15] J. Kaneko, The Gauss-Manin connection of the integral of the deformed difference product, Duke Math. J. 92 (1998), 355-379. MR 99h:32024

[16] S. Kobayashi, Differential geometry of complex vector bundles, Princeton Univ. Press, Princeton, NJ, 1987. MR 89e:53100

[17] P. Orlik, H. Terao, Arrangements of Hyperplanes, Grundlehren Math. Wiss., vol. 300, Springer-Verlag, Berlin, 1992. MR 94e:52014

[18] , Arrangements and Hypergeometric Integrals, MSJ Mem., vol. 9, Math. Soc. Japan, Tokyo, 2001. MR 2003a:32048

[19] R. Randell, Lattice-isotopic arrangements are topologically isomorphic, Proc. Amer. Math. Soc. 107 (1989), 555-559. MR 90a:57032

[20] V. Schechtman, H. Terao, A. Varchenko, Cohomology of local systems and the Kac-Kazhdan condition for singular vectors, J. Pure Appl. Algebra 100 (1995), 93-102. MR 96j:32047

[21] V. Schechtman and A. Varchenko, Arrangements of hyperplanes and Lie algebra homology, Invent. Math. 106 (1991), 139-194. MR 93b:17067 
[22] H. Terao, Moduli space of combinatorially equivalent arrangements of hyperplanes and logarithmic Gauss-Manin connections, Topology Appl. 118 (2002), 255-274. MR 2003e:32049

[23] A. Varchenko, Multidimensional Hypergeometric Functions and Representation Theory of Lie Algebras and Quantum Groups, Adv. Ser. Math. Phys., vol. 21, World Scientific, River Edge, NJ, 1995. MR 99i:32029 70803

Department of Mathematics, Louisiana State University, Baton Rouge, Louisiana

E-mail address: cohen@math.lsu.edu

URL: http://www. math.1su.edu/ cohen

Department of Mathematics, University of Wisconsin, Madison, Wisconsin 53706

E-mail address: orlik@math.wisc.edu 